\title{
Water-Mediated Interactions Enhance Alkaline Earth Cations Chelation in Neighboring Cavities of a Cytosine Quartet in DNA Quadruplex
}

\author{
Branislav Milovanović, ${ }^{1}$, Milena Petković ${ }^{1}$, Igor Popov, ${ }^{2,3}$ and Mihajlo Etinski ${ }^{1 *}$
}

\footnotetext{
${ }^{1}$ Faculty of Physical Chemistry, University of Belgrade, Studentski trg 12-16 11000 Belgrade, Serbia,

${ }^{2}$ Institute for Multidisciplinary Research, University of Belgrade, Kneza Višeslava 1, 11030 Belgrade, Serbia

${ }^{3}$ Institut of Physics, University of Belgrade, Pregrevica 118, 11080 Belgrade, Serbia

${ }^{*}$ To whom correspondence should be addressed; E-mail: etinski@ffh.bg.ac.rs
}

Supporting Information 
Table S1: The Cartesian coordinates of the structure 1 in $\AA$.

\begin{tabular}{cccc}
\hline \hline $\mathrm{C}$ & -0.509000038 & -4.561000098 & 1.782498135 \\
$\mathrm{C}$ & 0.341000025 & -5.646000179 & 1.698499131 \\
$\mathrm{C}$ & 0.038000001 & -3.258000000 & 1.789498139 \\
$\mathrm{C}$ & 2.206000169 & -4.427000089 & 1.735501131 \\
$\mathrm{C}$ & -1.771000135 & -6.278000230 & 1.714501129 \\
$\mathrm{H}$ & 1.917000148 & -2.387999931 & 1.648500126 \\
$\mathrm{H}$ & -0.182951746 & -7.691312057 & 1.528758157 \\
$\mathrm{H}$ & -2.638000202 & -6.922000279 & 1.714501129 \\
$\mathrm{H}$ & 3.908000300 & -3.324000002 & 1.883500143 \\
$\mathrm{H}$ & 4.130000316 & -5.076000139 & 1.845498142 \\
$\mathrm{~N}$ & 1.433000110 & -3.283000000 & 1.718499131 \\
$\mathrm{~N}$ & -0.472000034 & -6.739000266 & 1.626500127 \\
$\mathrm{~N}$ & 1.684000130 & -5.650000182 & 1.692498129 \\
$\mathrm{~N}$ & -1.833000139 & -4.972000130 & 1.798501137 \\
$\mathrm{~N}$ & 3.514000268 & -4.263000074 & 1.828500138 \\
$\mathrm{O}$ & -0.567000045 & -2.165999915 & 1.847501141 \\
$\mathrm{C}$ & 0.509000038 & 4.561000596 & 1.782498135 \\
$\mathrm{C}$ & -0.341000025 & 5.646000682 & 1.698499131 \\
$\mathrm{C}$ & -0.038000001 & 3.257999497 & 1.789498139 \\
$\mathrm{C}$ & -2.206000169 & 4.427000587 & 1.735501131 \\
$\mathrm{C}$ & 1.771000135 & 6.278000727 & 1.714501129 \\
$\mathrm{H}$ & -1.917000148 & 2.388000434 & 1.648500126 \\
$\mathrm{H}$ & 0.182951746 & 7.691312560 & 1.528758157 \\
$\mathrm{H}$ & 2.638000202 & 6.922001776 & 1.714501129 \\
$\mathrm{H}$ & -4.130000316 & 5.076000636 & 1.845498142 \\
$\mathrm{H}$ & -3.908000300 & 3.323999505 & 1.883500143 \\
$\mathrm{~N}$ & -1.433000110 & 3.283001503 & 1.718499131 \\
$\mathrm{~N}$ & 0.472000034 & 6.739000764 & 1.626500127 \\
$\mathrm{~N}$ & -1.684000130 & 5.649999680 & 1.692498129 \\
$\mathrm{~N}$ & 1.833000139 & 4.972000632 & 1.798501137 \\
$\mathrm{~N}$ & -3.514000268 & 4.263000577 & 1.828500138 \\
$\mathrm{O}$ & 0.567000045 & 2.166000417 & 1.847501141 \\
$\mathrm{C}$ & 3.008000231 & 3.450000513 & -1.557500118 \\
$\mathrm{C}$ & 2.955000224 & 4.826000619 & -1.384499105 \\
$\mathrm{C}$ & 1.797000137 & 2.731001458 & -1.685500130 \\
$\mathrm{C}$ & 0.743000058 & 4.951999627 & -1.523499114 \\
$\mathrm{C}$ & 5.039000383 & 4.085001559 & -1.357499106 \\
$\mathrm{H}$ & -0.234000020 & 3.146000492 & -1.693500131 \\
$\mathrm{H}$ & 4.593651218 & 6.162787669 & -1.096329342 \\
$\mathrm{H}$ & 6.117000465 & 4.083999562 & -1.291499098 \\
\hline & & &
\end{tabular}

Continued on next page 
Table S1 - Continued from previous page

\begin{tabular}{llrl}
\hline \hline $\mathrm{H}$ & -0.431000031 & 6.618000755 & -1.451501110 \\
$\mathrm{H}$ & -1.289000101 & 5.083000640 & -1.623500126 \\
$\mathrm{~N}$ & 0.689000055 & 3.576999523 & -1.638499126 \\
$\mathrm{~N}$ & 4.266000324 & 5.229999651 & -1.246501093 \\
$\mathrm{~N}$ & 1.880000144 & 5.623001681 & -1.385501107 \\
$\mathrm{~N}$ & 4.324000331 & 3.002000477 & -1.550500119 \\
$\mathrm{~N}$ & -0.415000034 & 5.600999676 & -1.533500115 \\
$\mathrm{O}$ & 1.646000123 & 1.507999365 & -1.847501141 \\
$\mathrm{C}$ & 1.547000119 & -4.242000072 & 5.063500385 \\
$\mathrm{C}$ & 2.750000212 & -4.887000124 & 5.280500401 \\
$\mathrm{C}$ & 1.541000117 & -2.846999969 & 4.809499369 \\
$\mathrm{C}$ & 3.994000302 & -3.033999979 & 5.129499392 \\
$\mathrm{C}$ & 1.081000083 & -6.306000231 & 5.365499412 \\
$\mathrm{H}$ & 2.921000221 & -1.312000848 & 4.747499365 \\
$\mathrm{H}$ & 3.098049958 & -6.948363990 & 5.638793563 \\
$\mathrm{H}$ & 0.541000042 & -7.233000304 & 5.490499418 \\
$\mathrm{H}$ & 6.005000460 & -2.817999965 & 5.372499411 \\
$\mathrm{H}$ & 5.105000391 & -1.327998850 & 5.068502385 \\
$\mathrm{~N}$ & 2.826000215 & -2.317999925 & 4.884500375 \\
$\mathrm{~N}$ & 2.452000188 & -6.205000223 & 5.465501418 \\
$\mathrm{~N}$ & 3.992000304 & -4.343000080 & 5.331498408 \\
$\mathrm{~N}$ & 0.500000040 & -5.160000142 & 5.103501390 \\
$\mathrm{~N}$ & 5.121000393 & -2.339999929 & 5.195500395 \\
$\mathrm{O}$ & 0.572000045 & -2.136999911 & 4.561502346 \\
$\mathrm{C}$ & 1.638000127 & -4.225000073 & -4.816501368 \\
$\mathrm{C}$ & 2.893000220 & -4.816000116 & -4.943500379 \\
$\mathrm{C}$ & 1.570000121 & -2.816999963 & -4.708501361 \\
$\mathrm{C}$ & 4.030000310 & -2.911999974 & -4.805501366 \\
$\mathrm{C}$ & 1.272000097 & -6.319000232 & -5.062501388 \\
$\mathrm{H}$ & 2.860000218 & -1.209998843 & -4.758500362 \\
$\mathrm{H}$ & 3.337604207 & -6.879602704 & -5.214335528 \\
$\mathrm{H}$ & 0.773000059 & -7.270000303 & -5.177501394 \\
$\mathrm{H}$ & 6.054000463 & -2.644999952 & -4.774501364 \\
$\mathrm{H}$ & 5.086000387 & -1.170999840 & -4.662499353 \\
$\mathrm{~N}$ & 2.822000217 & -2.228999921 & -4.757501365 \\
$\mathrm{~N}$ & 2.654000204 & -6.159000220 & -5.098500390 \\
$\mathrm{~N}$ & 4.099000314 & -4.236000075 & -4.918500373 \\
$\mathrm{~N}$ & 0.641000048 & -5.175000148 & -4.871501374 \\
$\mathrm{~N}$ & 5.143000392 & -2.185999915 & -4.742499365 \\
& 0.535000040 & -2.123999910 & -4.583501350 \\
& 3.450000264 & -3.007999982 & -1.557500118 \\
\hline & & Continued on next page
\end{tabular}


Table S1 - Continued from previous page

\begin{tabular}{cccc}
\hline $\mathrm{C}$ & 4.826000370 & -2.954999976 & -1.384499105 \\
$\mathrm{C}$ & 2.731000209 & -1.796998888 & -1.685500130 \\
$\mathrm{C}$ & 4.952000378 & -0.742999804 & -1.523499114 \\
$\mathrm{C}$ & 4.085000310 & -5.039000134 & -1.357499106 \\
$\mathrm{H}$ & 3.146000238 & 0.234001269 & -1.693500131 \\
$\mathrm{H}$ & 6.162788441 & -4.593651033 & -1.096329596 \\
$\mathrm{H}$ & 4.084000314 & -6.117000216 & -1.291499098 \\
$\mathrm{H}$ & 6.618000507 & 0.430999285 & -1.451501110 \\
$\mathrm{H}$ & 5.083000386 & 1.288999349 & -1.623500126 \\
$\mathrm{~N}$ & 3.577000275 & -0.688999801 & -1.638499126 \\
$\mathrm{~N}$ & 5.230000397 & -4.266000076 & -1.246501093 \\
$\mathrm{~N}$ & 5.623000432 & -1.879998895 & -1.385501107 \\
$\mathrm{~N}$ & 3.002000229 & -4.324000082 & -1.550500119 \\
$\mathrm{~N}$ & 5.601000428 & 0.415001283 & -1.533500115 \\
$\mathrm{O}$ & 1.508000116 & -1.645999874 & -1.847501141 \\
$\mathrm{C}$ & 4.225000322 & 1.638000376 & -4.816501368 \\
$\mathrm{C}$ & 4.816000370 & 2.892999473 & -4.943500379 \\
$\mathrm{C}$ & 2.817000217 & 1.569999369 & -4.708501361 \\
$\mathrm{C}$ & 2.912000223 & 4.030000559 & -4.805501366 \\
$\mathrm{C}$ & 6.319000481 & 1.272001345 & -5.062501388 \\
$\mathrm{H}$ & 1.210000092 & 2.860000467 & -4.758500362 \\
$\mathrm{H}$ & 6.879602465 & 3.337605985 & -5.214335448 \\
$\mathrm{H}$ & 7.270000557 & 0.773000307 & -5.177501394 \\
$\mathrm{H}$ & 1.171000089 & 5.086000636 & -4.662499353 \\
$\mathrm{H}$ & 2.645000201 & 6.054001712 & -4.774501364 \\
$\mathrm{~N}$ & 2.229000170 & 2.822000466 & -4.757501365 \\
$\mathrm{~N}$ & 6.159000469 & 2.654001453 & -5.098500390 \\
$\mathrm{~N}$ & 4.236000324 & 4.099001563 & -4.918500373 \\
$\mathrm{~N}$ & 5.175000396 & 0.641000297 & -4.871501374 \\
$\mathrm{~N}$ & 2.186000169 & 5.142999641 & -4.742499365 \\
$\mathrm{O}$ & 2.124000164 & 0.535000289 & -4.583501350 \\
$\mathrm{C}$ & 4.242000326 & 1.547001368 & 5.063500385 \\
$\mathrm{C}$ & 4.887000372 & 2.750000461 & 5.280500401 \\
$\mathrm{C}$ & 2.847000217 & 1.541000366 & 4.809499369 \\
$\mathrm{C}$ & 3.034000233 & 3.993999556 & 5.129499392 \\
$\mathrm{C}$ & 6.306000479 & 1.081001332 & 5.365499412 \\
$\mathrm{H}$ & 1.312000102 & 2.920999475 & 4.747499365 \\
$\mathrm{H}$ & 6.948364408 & 3.098048958 & 5.638793722 \\
$\mathrm{H}$ & 7.233000553 & 0.541000291 & 5.490499418 \\
$\mathrm{H}$ & 1.328000099 & 5.104999639 & 5.068502385 \\
& 2.818000214 & 6.005001708 & 5.372499411 \\
\hline & & Continued on next page
\end{tabular}


Table S1 - Continued from previous page

\begin{tabular}{cccc}
\hline \hline $\mathrm{N}$ & 2.318000179 & 2.826000464 & 4.884500375 \\
$\mathrm{~N}$ & 6.205000471 & 2.451999437 & 5.465501418 \\
$\mathrm{~N}$ & 4.343000334 & 3.992000552 & 5.331498408 \\
$\mathrm{~N}$ & 5.160000396 & 0.500000289 & 5.103501390 \\
$\mathrm{~N}$ & 2.340000178 & 5.121000642 & 5.195500395 \\
$\mathrm{O}$ & 2.137000165 & 0.572000293 & 4.561502346 \\
$\mathrm{C}$ & 4.561000347 & -0.508998789 & 1.782498135 \\
$\mathrm{C}$ & 5.646000433 & 0.340999274 & 1.698499131 \\
$\mathrm{C}$ & 3.258000248 & 0.038000255 & 1.789498139 \\
$\mathrm{C}$ & 4.427000338 & 2.206001418 & 1.735501131 \\
$\mathrm{C}$ & 6.278000478 & -1.770999886 & 1.714501129 \\
$\mathrm{H}$ & 2.388000185 & 1.916999397 & 1.648500126 \\
$\mathrm{H}$ & 7.691312216 & -0.182951201 & 1.528758178 \\
$\mathrm{H}$ & 6.922000528 & -2.637999954 & 1.714501129 \\
$\mathrm{H}$ & 3.324000256 & 3.908001549 & 1.883500143 \\
$\mathrm{H}$ & 5.076000387 & 4.130001565 & 1.845498142 \\
$\mathrm{~N}$ & 3.283000248 & 1.433000359 & 1.718499131 \\
$\mathrm{~N}$ & 6.739000515 & -0.471999785 & 1.626500127 \\
$\mathrm{~N}$ & 5.650000431 & 1.684000378 & 1.692498129 \\
$\mathrm{~N}$ & 4.972000378 & -1.833000891 & 1.798501137 \\
$\mathrm{~N}$ & 4.263000323 & 3.514000517 & 1.828500138 \\
$\mathrm{O}$ & 2.166000163 & -0.566999791 & 1.847501141 \\
$\mathrm{C}$ & -3.008000231 & -3.450000016 & -1.557500118 \\
$\mathrm{C}$ & -2.955000224 & -4.826000116 & -1.384499105 \\
$\mathrm{C}$ & -1.797000137 & -2.730999960 & -1.685500130 \\
$\mathrm{C}$ & -0.743000058 & -4.952000129 & -1.523499114 \\
$\mathrm{C}$ & -5.039000383 & -4.085000062 & -1.357499106 \\
$\mathrm{H}$ & 0.234000020 & -3.145999989 & -1.693500131 \\
$\mathrm{H}$ & -4.593651282 & -6.162788193 & -1.096329596 \\
$\mathrm{H}$ & -6.117000465 & -4.084000060 & -1.291499098 \\
$\mathrm{H}$ & 0.431000031 & -6.618000258 & -1.451501110 \\
$\mathrm{H}$ & 1.289000101 & -5.083000138 & -1.623500126 \\
$\mathrm{~N}$ & -0.689000055 & -3.577000021 & -1.638499126 \\
$\mathrm{~N}$ & -4.266000324 & -5.230000148 & -1.246501093 \\
$\mathrm{~N}$ & -1.880000144 & -5.623000178 & -1.385501107 \\
$\mathrm{~N}$ & -4.324000331 & -3.001999980 & -1.550500119 \\
$\mathrm{~N}$ & 0.415000034 & -5.601000179 & -1.533500115 \\
$\mathrm{O}$ & -1.646000123 & -1.507999867 & -1.847501141 \\
$\mathrm{C}$ & -1.547000119 & 4.242000575 & 5.063500385 \\
$\mathrm{C}$ & -2.750000212 & 4.887001621 & 5.280500401 \\
& -1.541000117 & 2.847000466 & 4.809499369 \\
\hline & & Continued & on next page
\end{tabular}


Table S1 - Continued from previous page

\begin{tabular}{ccrc}
\hline \hline $\mathrm{C}$ & -3.994000302 & 3.034000482 & 5.129499392 \\
$\mathrm{C}$ & -1.081000083 & 6.305999733 & 5.365499412 \\
$\mathrm{H}$ & -2.921000221 & 1.312000351 & 4.747499365 \\
$\mathrm{H}$ & -3.098049625 & 6.948364773 & 5.638793568 \\
$\mathrm{H}$ & -0.541000042 & 7.232999801 & 5.490499418 \\
$\mathrm{H}$ & -6.005000460 & 2.818000463 & 5.372499411 \\
$\mathrm{H}$ & -5.105000391 & 1.328001353 & 5.068502385 \\
$\mathrm{~N}$ & -2.826000215 & 2.318000428 & 4.884500375 \\
$\mathrm{~N}$ & -2.452000188 & 6.205000725 & 5.465501418 \\
$\mathrm{~N}$ & -3.992000304 & 4.343000583 & 5.331498408 \\
$\mathrm{~N}$ & -0.500000040 & 5.160000645 & 5.103501390 \\
$\mathrm{~N}$ & -5.121000393 & 2.340000427 & 5.195500395 \\
$\mathrm{O}$ & -0.572000045 & 2.137001414 & 4.561502346 \\
$\mathrm{C}$ & -1.638000127 & 4.225000571 & -4.816501368 \\
$\mathrm{C}$ & -2.893000220 & 4.816000619 & -4.943500379 \\
$\mathrm{C}$ & -1.570000121 & 2.816999466 & -4.708501361 \\
$\mathrm{C}$ & -4.030000310 & 2.912000472 & -4.805501366 \\
$\mathrm{C}$ & -1.272000097 & 6.319000735 & -5.062501388 \\
$\mathrm{H}$ & -2.860000218 & 1.210001341 & -4.758500362 \\
$\mathrm{H}$ & -3.337604207 & 6.879603206 & -5.214335528 \\
$\mathrm{H}$ & -0.773000059 & 7.270000806 & -5.177501394 \\
$\mathrm{H}$ & -6.054000463 & 2.645000450 & -4.774501364 \\
$\mathrm{H}$ & -5.086000387 & 1.170999337 & -4.662499353 \\
$\mathrm{~N}$ & -2.822000217 & 2.229000419 & -4.757501365 \\
$\mathrm{~N}$ & -2.654000204 & 6.159000718 & -5.098500390 \\
$\mathrm{~N}$ & -4.099000314 & 4.236000573 & -4.918500373 \\
$\mathrm{~N}$ & -0.641000048 & 5.175001645 & -4.871501374 \\
$\mathrm{~N}$ & -5.143000392 & 2.186000418 & -4.742499365 \\
$\mathrm{O}$ & -0.535000040 & 2.124000413 & -4.583501350 \\
$\mathrm{C}$ & -3.450000264 & 3.007999479 & -1.557500118 \\
$\mathrm{C}$ & -4.826000370 & 2.955000478 & -1.384499105 \\
$\mathrm{C}$ & -2.731000209 & 1.797001386 & -1.685500130 \\
$\mathrm{C}$ & -4.952000378 & 0.743000307 & -1.523499114 \\
$\mathrm{C}$ & -4.085000310 & 5.038999637 & -1.357499106 \\
$\mathrm{H}$ & -3.146000238 & -0.233999766 & -1.693500131 \\
$\mathrm{H}$ & -6.162788341 & 4.593651811 & -1.096329623 \\
$\mathrm{H}$ & -4.084000314 & 6.117000719 & -1.291499098 \\
$\mathrm{H}$ & -6.618000507 & -0.430999782 & -1.451501110 \\
$\mathrm{~N}$ & -5.083000386 & -1.288999847 & -1.623500126 \\
& -5.230000397 & 0.689001304 & -1.638499126 \\
& 4.266000578 & -1.246501093 \\
\hline
\end{tabular}


Table S1 - Continued from previous page

\begin{tabular}{llll}
\hline \hline $\mathrm{N}$ & -5.623000432 & 1.880001393 & -1.385501107 \\
$\mathrm{~N}$ & -3.002000229 & 4.323999580 & -1.550500119 \\
$\mathrm{~N}$ & -5.601000428 & -0.414998780 & -1.533500115 \\
$\mathrm{O}$ & -1.508000116 & 1.646000377 & -1.847501141 \\
$\mathrm{C}$ & -4.225000322 & -1.638000874 & -4.816501368 \\
$\mathrm{C}$ & -4.816000370 & -2.892999971 & -4.943500379 \\
$\mathrm{C}$ & -2.817000217 & -1.569999872 & -4.708501361 \\
$\mathrm{C}$ & -2.912000223 & -4.030000056 & -4.805501366 \\
$\mathrm{C}$ & -6.319000481 & -1.271999848 & -5.062501388 \\
$\mathrm{H}$ & -1.210000092 & -2.859999970 & -4.758500362 \\
$\mathrm{H}$ & -6.879602958 & -3.337603953 & -5.214335528 \\
$\mathrm{H}$ & -7.270000557 & -0.772998810 & -5.177501394 \\
$\mathrm{H}$ & -1.171000089 & -5.086000139 & -4.662499353 \\
$\mathrm{H}$ & -2.645000201 & -6.054000214 & -4.774501364 \\
$\mathrm{~N}$ & -2.229000170 & -2.821999963 & -4.757501365 \\
$\mathrm{~N}$ & -6.159000469 & -2.653999950 & -5.098500390 \\
$\mathrm{~N}$ & -4.236000324 & -4.099000065 & -4.918500373 \\
$\mathrm{~N}$ & -5.175000396 & -0.640998799 & -4.871501374 \\
$\mathrm{~N}$ & -2.186000169 & -5.143000143 & -4.742499365 \\
$\mathrm{O}$ & -2.124000164 & -0.534999792 & -4.583501350 \\
$\mathrm{C}$ & -4.242000326 & -1.546998871 & 5.063500385 \\
$\mathrm{C}$ & -4.887000372 & -2.749999958 & 5.280500401 \\
$\mathrm{C}$ & -2.847000217 & -1.541000869 & 4.809499369 \\
$\mathrm{C}$ & -3.034000233 & -3.994000054 & 5.129499392 \\
$\mathrm{C}$ & -6.306000479 & -1.080999834 & 5.365499412 \\
$\mathrm{H}$ & -1.312000102 & -2.920999972 & 4.747499365 \\
$\mathrm{H}$ & -6.948364239 & -3.098049704 & 5.638793563 \\
$\mathrm{H}$ & -7.233000553 & -0.541000794 & 5.490499418 \\
$\mathrm{H}$ & -1.328000099 & -5.105000142 & 5.068502385 \\
$\mathrm{H}$ & -2.818000214 & -6.005000211 & 5.372499411 \\
$\mathrm{~N}$ & -2.318000179 & -2.825999966 & 4.884500375 \\
$\mathrm{~N}$ & -6.205000471 & -2.451999940 & 5.465501418 \\
$\mathrm{~N}$ & -4.343000334 & -3.992000055 & 5.331498408 \\
$\mathrm{~N}$ & -5.160000396 & -0.499999786 & 5.103501390 \\
$\mathrm{~N}$ & -2.340000178 & -5.121000139 & 5.195500395 \\
$\mathrm{O}$ & -2.137000165 & -0.572000796 & 4.561502346 \\
$\mathrm{C}$ & -4.561000347 & 0.509000287 & 1.782498135 \\
$\mathrm{C}$ & -5.646000433 & -0.340999777 & 1.698499131 \\
$\mathrm{C}$ & -3.258000248 & -0.037999753 & 1.789498139 \\
& -4.427000338 & -2.205999920 & 1.735501131 \\
& -6.278000478 & 1.771000383 & 1.714501129 \\
\hline & & Continued & on next page
\end{tabular}


Table S1 - Continued from previous page

\begin{tabular}{cccc}
\hline \hline $\mathrm{H}$ & -2.388000185 & -1.916999894 & 1.648500126 \\
$\mathrm{H}$ & -7.691312306 & 0.182951995 & 1.528758157 \\
$\mathrm{H}$ & -6.922000528 & 2.638000451 & 1.714501129 \\
$\mathrm{H}$ & -3.324000256 & -3.908000046 & 1.883500143 \\
$\mathrm{H}$ & -5.076000387 & -4.130000067 & 1.845498142 \\
$\mathrm{~N}$ & -3.283000248 & -1.433000862 & 1.718499131 \\
$\mathrm{~N}$ & -6.739000515 & 0.472000288 & 1.626500127 \\
$\mathrm{~N}$ & -5.650000431 & -1.683999881 & 1.692498129 \\
$\mathrm{~N}$ & -4.972000378 & 1.833000388 & 1.798501137 \\
$\mathrm{~N}$ & -4.263000323 & -3.514000019 & 1.828500138 \\
$\mathrm{O}$ & -2.166000163 & 0.566999293 & 1.847501141 \\
$\mathrm{Sr}$ & 0.000000011 & 0.000000249 & 3.181500242 \\
$\mathrm{Sr}$ & 0.000000021 & 0.000000259 & -3.189500243 \\
\hline
\end{tabular}


Table S2: The Cartesian coordinates of the structure $\mathbf{2}$ in $\AA$.

\begin{tabular}{cccc}
\hline $\mathrm{C}$ & -0.509000038 & -4.561000098 & 3.629999276 \\
$\mathrm{C}$ & 0.341000025 & -5.646000179 & 3.546000272 \\
$\mathrm{C}$ & 0.038000001 & -3.258000000 & 3.636999280 \\
$\mathrm{C}$ & 2.206000169 & -4.427000089 & 3.583002272 \\
$\mathrm{C}$ & -1.771000135 & -6.278000230 & 3.562002269 \\
$\mathrm{H}$ & 1.917000148 & -2.387999931 & 3.496001267 \\
$\mathrm{H}$ & -0.182951746 & -7.691312057 & 3.376259298 \\
$\mathrm{H}$ & -2.638000202 & -6.922000279 & 3.562002269 \\
$\mathrm{H}$ & 3.908000300 & -3.324000002 & 3.731001284 \\
$\mathrm{H}$ & 4.130000316 & -5.076000139 & 3.692999283 \\
$\mathrm{~N}$ & 1.433000110 & -3.283000000 & 3.566000272 \\
$\mathrm{~N}$ & -0.472000034 & -6.739000266 & 3.474001268 \\
$\mathrm{~N}$ & 1.684000130 & -5.650000182 & 3.539999270 \\
$\mathrm{~N}$ & -1.833000139 & -4.972000130 & 3.646002278 \\
$\mathrm{~N}$ & 3.514000268 & -4.263000074 & 3.676001278 \\
$\mathrm{O}$ & -0.567000045 & -2.165999915 & 3.695002282 \\
$\mathrm{C}$ & 0.509000038 & 4.561000596 & 3.629999276 \\
$\mathrm{C}$ & -0.341000025 & 5.646000682 & 3.546000272 \\
$\mathrm{C}$ & -0.038000001 & 3.257999497 & 3.636999280 \\
$\mathrm{C}$ & -2.206000169 & 4.427000587 & 3.583002272 \\
$\mathrm{C}$ & 1.771000135 & 6.278000727 & 3.562002269 \\
$\mathrm{H}$ & -1.917000148 & 2.388000434 & 3.496001267 \\
$\mathrm{H}$ & 0.182951746 & 7.691312560 & 3.376259298 \\
$\mathrm{H}$ & 2.638000202 & 6.922001776 & 3.562002269 \\
$\mathrm{H}$ & -4.130000316 & 5.076000636 & 3.692999283 \\
$\mathrm{H}$ & -3.908000300 & 3.323999505 & 3.731001284 \\
$\mathrm{~N}$ & -1.433000110 & 3.283001503 & 3.566000272 \\
$\mathrm{~N}$ & 0.472000034 & 6.739000764 & 3.474001268 \\
$\mathrm{~N}$ & -1.684000130 & 5.649999680 & 3.539999270 \\
$\mathrm{~N}$ & 1.833000139 & 4.972000632 & 3.646002278 \\
$\mathrm{~N}$ & -3.514000268 & 4.263000577 & 3.676001278 \\
$\mathrm{O}$ & 0.567000045 & 2.166000417 & 3.695002282 \\
$\mathrm{C}$ & 3.008000231 & 3.450000513 & 0.290001023 \\
$\mathrm{C}$ & 2.955000224 & 4.826000619 & 0.463002036 \\
$\mathrm{C}$ & 1.797000137 & 2.731001458 & 0.162001011 \\
$\mathrm{C}$ & 0.743000058 & 4.951999627 & 0.324002027 \\
$\mathrm{C}$ & 5.039000383 & 4.085001559 & 0.490002035 \\
$\mathrm{H}$ & -0.234000020 & 3.146000492 & 0.154001010 \\
$\mathrm{H}$ & 4.593651218 & 6.162787669 & 0.751171799 \\
& 6.117000465 & 4.083999562 & 0.556002043 \\
\hline & & &
\end{tabular}

Continued on next page 
Table S2 - Continued from previous page

\begin{tabular}{llll}
\hline \hline $\mathrm{H}$ & -0.431000031 & 6.618000755 & 0.396000031 \\
$\mathrm{H}$ & -1.289000101 & 5.083000640 & 0.224001015 \\
$\mathrm{~N}$ & 0.689000055 & 3.576999523 & 0.209002015 \\
$\mathrm{~N}$ & 4.266000324 & 5.229999651 & 0.601000048 \\
$\mathrm{~N}$ & 1.880000144 & 5.623001681 & 0.462000033 \\
$\mathrm{~N}$ & 4.324000331 & 3.002000477 & 0.297001022 \\
$\mathrm{~N}$ & -0.415000034 & 5.600999676 & 0.314001026 \\
$\mathrm{O}$ & 1.646000123 & 1.507999365 & 0.000000000 \\
$\mathrm{C}$ & 1.547000119 & -4.242000072 & 6.911001526 \\
$\mathrm{C}$ & 2.750000212 & -4.887000124 & 7.128001542 \\
$\mathrm{C}$ & 1.541000117 & -2.846999969 & 6.657000510 \\
$\mathrm{C}$ & 3.994000302 & -3.033999979 & 6.977000533 \\
$\mathrm{C}$ & 1.081000083 & -6.306000231 & 7.213000553 \\
$\mathrm{H}$ & 2.921000221 & -1.312000848 & 6.595000506 \\
$\mathrm{H}$ & 3.098049958 & -6.948363990 & 7.486294704 \\
$\mathrm{H}$ & 0.541000042 & -7.233000304 & 7.338000559 \\
$\mathrm{H}$ & 6.005000460 & -2.817999965 & 7.220000552 \\
$\mathrm{H}$ & 5.105000391 & -1.327998850 & 6.916003526 \\
$\mathrm{~N}$ & 2.826000215 & -2.317999925 & 6.732001516 \\
$\mathrm{~N}$ & 2.452000188 & -6.205000223 & 7.313002559 \\
$\mathrm{~N}$ & 3.992000304 & -4.343000080 & 7.178999549 \\
$\mathrm{~N}$ & 0.500000040 & -5.160000142 & 6.951002531 \\
$\mathrm{~N}$ & 5.121000393 & -2.339999929 & 7.043001536 \\
$\mathrm{O}$ & 0.572000045 & -2.136999911 & 6.409003487 \\
$\mathrm{C}$ & 1.638000127 & -4.225000073 & -2.969000227 \\
$\mathrm{C}$ & 2.893000220 & -4.816000116 & -3.095999238 \\
$\mathrm{C}$ & 1.570000121 & -2.816999963 & -2.861000220 \\
$\mathrm{C}$ & 4.030000310 & -2.911999974 & -2.958000225 \\
$\mathrm{C}$ & 1.272000097 & -6.319000232 & -3.215000247 \\
$\mathrm{H}$ & 2.860000218 & -1.209998843 & -2.910999221 \\
$\mathrm{H}$ & 3.337604207 & -6.879602704 & -3.366834387 \\
$\mathrm{H}$ & 0.773000059 & -7.270000303 & -3.330000253 \\
$\mathrm{H}$ & 6.054000463 & -2.644999952 & -2.927000223 \\
$\mathrm{H}$ & 5.086000387 & -1.170999840 & -2.814998213 \\
$\mathrm{~N}$ & 2.822000217 & -2.228999921 & -2.910000224 \\
$\mathrm{~N}$ & 2.654000204 & -6.159000220 & -3.250999249 \\
$\mathrm{~N}$ & 4.099000314 & -4.236000075 & -3.070999232 \\
$\mathrm{~N}$ & 0.641000048 & -5.175000148 & -3.024000233 \\
$\mathrm{~N}$ & 5.143000392 & -2.185999915 & -2.894998224 \\
& 0.535000040 & -2.123999910 & -2.736000209 \\
& 3.450000264 & -3.007999982 & 0.290001023 \\
\hline & & &
\end{tabular}


Table S2 - Continued from previous page

\begin{tabular}{lccc}
\hline \hline $\mathrm{C}$ & 4.826000370 & -2.954999976 & 0.463002036 \\
$\mathrm{C}$ & 2.731000209 & -1.796998888 & 0.162001011 \\
$\mathrm{C}$ & 4.952000378 & -0.742999804 & 0.324002027 \\
$\mathrm{C}$ & 4.085000310 & -5.039000134 & 0.490002035 \\
$\mathrm{H}$ & 3.146000238 & 0.234001269 & 0.154001010 \\
$\mathrm{H}$ & 6.162788441 & -4.593651033 & 0.751171545 \\
$\mathrm{H}$ & 4.084000314 & -6.117000216 & 0.556002043 \\
$\mathrm{H}$ & 6.618000507 & 0.430999285 & 0.396000031 \\
$\mathrm{H}$ & 5.083000386 & 1.288999349 & 0.224001015 \\
$\mathrm{~N}$ & 3.577000275 & -0.688999801 & 0.209002015 \\
$\mathrm{~N}$ & 5.230000397 & -4.266000076 & 0.601000048 \\
$\mathrm{~N}$ & 5.623000432 & -1.879998895 & 0.462000033 \\
$\mathrm{~N}$ & 3.002000229 & -4.324000082 & 0.297001022 \\
$\mathrm{~N}$ & 5.601000428 & 0.415001283 & 0.314001026 \\
$\mathrm{O}$ & 1.508000116 & -1.645999874 & 0.000000000 \\
$\mathrm{C}$ & 4.225000322 & 1.638000376 & -2.969000227 \\
$\mathrm{C}$ & 4.816000370 & 2.892999473 & -3.095999238 \\
$\mathrm{C}$ & 2.817000217 & 1.569999369 & -2.861000220 \\
$\mathrm{C}$ & 2.912000223 & 4.030000559 & -2.958000225 \\
$\mathrm{C}$ & 6.319000481 & 1.272001345 & -3.215000247 \\
$\mathrm{H}$ & 1.210000092 & 2.860000467 & -2.910999221 \\
$\mathrm{H}$ & 6.879602465 & 3.337605985 & -3.366834307 \\
$\mathrm{H}$ & 7.270000557 & 0.773000307 & -3.330000253 \\
$\mathrm{H}$ & 1.171000089 & 5.086000636 & -2.814998213 \\
$\mathrm{H}$ & 2.645000201 & 6.054001712 & -2.927000223 \\
$\mathrm{~N}$ & 2.229000170 & 2.822000466 & -2.910000224 \\
$\mathrm{~N}$ & 6.159000469 & 2.654001453 & -3.250999249 \\
$\mathrm{~N}$ & 4.236000324 & 4.099001563 & -3.070999232 \\
$\mathrm{~N}$ & 5.175000396 & 0.641000297 & -3.024000233 \\
$\mathrm{~N}$ & 2.186000169 & 5.142999641 & -2.894998224 \\
$\mathrm{O}$ & 2.124000164 & 0.535000289 & -2.736000209 \\
$\mathrm{C}$ & 4.242000326 & 1.547001368 & 6.911001526 \\
$\mathrm{C}$ & 4.887000372 & 2.750000461 & 7.128001542 \\
$\mathrm{C}$ & 2.847000217 & 1.541000366 & 6.657000510 \\
$\mathrm{C}$ & 3.034000233 & 3.993999556 & 6.977000533 \\
$\mathrm{C}$ & 6.306000479 & 1.081001332 & 7.213000553 \\
$\mathrm{H}$ & 1.312000102 & 2.920999475 & 6.595000506 \\
$\mathrm{H}$ & 6.948364408 & 3.098048958 & 7.486294863 \\
$\mathrm{H}$ & 7.233000553 & 0.541000291 & 7.338000559 \\
& 2.818000214 & 6.005001708 & 7.220000552 \\
\hline & & & \\
$\mathrm{H}$ & &
\end{tabular}


Table S2 - Continued from previous page

\begin{tabular}{cccc}
\hline \hline $\mathrm{N}$ & 2.318000179 & 2.826000464 & 6.732001516 \\
$\mathrm{~N}$ & 6.205000471 & 2.451999437 & 7.313002559 \\
$\mathrm{~N}$ & 4.343000334 & 3.992000552 & 7.178999549 \\
$\mathrm{~N}$ & 5.160000396 & 0.500000289 & 6.951002531 \\
$\mathrm{~N}$ & 2.340000178 & 5.121000642 & 7.043001536 \\
$\mathrm{O}$ & 2.137000165 & 0.572000293 & 6.409003487 \\
$\mathrm{C}$ & 4.561000347 & -0.508998789 & 3.629999276 \\
$\mathrm{C}$ & 5.646000433 & 0.340999274 & 3.546000272 \\
$\mathrm{C}$ & 3.258000248 & 0.038000255 & 3.636999280 \\
$\mathrm{C}$ & 4.427000338 & 2.206001418 & 3.583002272 \\
$\mathrm{C}$ & 6.278000478 & -1.770999886 & 3.562002269 \\
$\mathrm{H}$ & 2.388000185 & 1.916999397 & 3.496001267 \\
$\mathrm{H}$ & 7.691312216 & -0.182951201 & 3.376259319 \\
$\mathrm{H}$ & 6.922000528 & -2.637999954 & 3.562002269 \\
$\mathrm{H}$ & 3.324000256 & 3.908001549 & 3.731001284 \\
$\mathrm{H}$ & 5.076000387 & 4.130001565 & 3.692999283 \\
$\mathrm{~N}$ & 3.283000248 & 1.433000359 & 3.566000272 \\
$\mathrm{~N}$ & 6.739000515 & -0.471999785 & 3.474001268 \\
$\mathrm{~N}$ & 5.650000431 & 1.684000378 & 3.539999270 \\
$\mathrm{~N}$ & 4.972000378 & -1.833000891 & 3.646002278 \\
$\mathrm{~N}$ & 4.263000323 & 3.514000517 & 3.676001278 \\
$\mathrm{O}$ & 2.166000163 & -0.566999791 & 3.695002282 \\
$\mathrm{C}$ & -3.008000231 & -3.450000016 & 0.290001023 \\
$\mathrm{C}$ & -2.955000224 & -4.826000116 & 0.463002036 \\
$\mathrm{C}$ & -1.797000137 & -2.730999960 & 0.162001011 \\
$\mathrm{C}$ & -0.743000058 & -4.952000129 & 0.324002027 \\
$\mathrm{C}$ & -5.039000383 & -4.085000062 & 0.490002035 \\
$\mathrm{H}$ & 0.234000020 & -3.145999989 & 0.154001010 \\
$\mathrm{H}$ & -4.593651282 & -6.162788193 & 0.751171545 \\
$\mathrm{H}$ & -6.117000465 & -4.084000060 & 0.556002043 \\
$\mathrm{H}$ & 0.431000031 & -6.618000258 & 0.396000031 \\
$\mathrm{H}$ & 1.289000101 & -5.083000138 & 0.224001015 \\
$\mathrm{~N}$ & -0.689000055 & -3.577000021 & 0.209002015 \\
$\mathrm{~N}$ & -4.266000324 & -5.230000148 & 0.601000048 \\
$\mathrm{~N}$ & -1.880000144 & -5.623000178 & 0.462000033 \\
$\mathrm{~N}$ & -4.324000331 & -3.001999980 & 0.297001022 \\
$\mathrm{~N}$ & 0.415000034 & -5.601000179 & 0.314001026 \\
$\mathrm{O}$ & -1.646000123 & -1.507999867 & 0.000000000 \\
$\mathrm{C}$ & -1.547000119 & 4.242000575 & 6.911001526 \\
$\mathrm{C}$ & -2.750000212 & 4.887001621 & 7.128001542 \\
& -1.541000117 & 2.847000466 & 6.657000510 \\
\hline & & Continued & on next page
\end{tabular}


Table S2 - Continued from previous page

\begin{tabular}{crrr}
\hline $\mathrm{C}$ & -3.994000302 & 3.034000482 & 6.977000533 \\
$\mathrm{C}$ & -1.081000083 & 6.305999733 & 7.213000553 \\
$\mathrm{H}$ & -2.921000221 & 1.312000351 & 6.595000506 \\
$\mathrm{H}$ & -3.098049625 & 6.948364773 & 7.486294709 \\
$\mathrm{H}$ & -0.541000042 & 7.232999801 & 7.338000559 \\
$\mathrm{H}$ & -6.005000460 & 2.818000463 & 7.220000552 \\
$\mathrm{H}$ & -5.105000391 & 1.328001353 & 6.916003526 \\
$\mathrm{~N}$ & -2.826000215 & 2.318000428 & 6.732001516 \\
$\mathrm{~N}$ & -2.452000188 & 6.205000725 & 7.313002559 \\
$\mathrm{~N}$ & -3.992000304 & 4.343000583 & 7.178999549 \\
$\mathrm{~N}$ & -0.500000040 & 5.160000645 & 6.951002531 \\
$\mathrm{~N}$ & -5.121000393 & 2.340000427 & 7.043001536 \\
$\mathrm{O}$ & -0.572000045 & 2.137001414 & 6.409003487 \\
$\mathrm{C}$ & -1.638000127 & 4.225000571 & -2.969000227 \\
$\mathrm{C}$ & -2.893000220 & 4.816000619 & -3.095999238 \\
$\mathrm{C}$ & -1.570000121 & 2.816999466 & -2.861000220 \\
$\mathrm{C}$ & -4.030000310 & 2.912000472 & -2.958000225 \\
$\mathrm{C}$ & -1.272000097 & 6.319000735 & -3.215000247 \\
$\mathrm{H}$ & -2.860000218 & 1.210001341 & -2.910999221 \\
$\mathrm{H}$ & -3.337604207 & 6.879603206 & -3.366834387 \\
$\mathrm{H}$ & -0.773000059 & 7.270000806 & -3.330000253 \\
$\mathrm{H}$ & -6.054000463 & 2.645000450 & -2.927000223 \\
$\mathrm{H}$ & -5.086000387 & 1.170999337 & -2.814998213 \\
$\mathrm{~N}$ & -2.822000217 & 2.229000419 & -2.910000224 \\
$\mathrm{~N}$ & -2.654000204 & 6.159000718 & -3.250999249 \\
$\mathrm{~N}$ & -4.099000314 & 4.236000573 & -3.070999232 \\
$\mathrm{~N}$ & -0.641000048 & 5.175001645 & -3.024000233 \\
$\mathrm{~N}$ & -5.143000392 & 2.186000418 & -2.894998224 \\
$\mathrm{O}$ & -0.535000040 & 2.124000413 & -2.736000209 \\
$\mathrm{C}$ & -3.450000264 & 3.007999479 & 0.290001023 \\
$\mathrm{C}$ & -4.826000370 & 2.955000478 & 0.463002036 \\
$\mathrm{C}$ & -2.731000209 & 1.797001386 & 0.162001011 \\
$\mathrm{C}$ & -4.952000378 & 0.743000307 & 0.324002027 \\
$\mathrm{C}$ & -4.085000310 & 5.038999637 & 0.490002035 \\
$\mathrm{H}$ & -3.146000238 & -0.233999766 & 0.154001010 \\
$\mathrm{H}$ & -6.162788341 & 4.593651811 & 0.751171518 \\
$\mathrm{H}$ & -4.084000314 & 6.117000719 & 0.556002043 \\
$\mathrm{H}$ & -6.618000507 & -0.430999782 & 0.396000031 \\
$\mathrm{H}$ & -5.083000386 & -1.288999847 & 0.224001015 \\
& -3.577000275 & 0.689001304 & 0.209002015 \\
& -5.230000397 & 4.266000578 & 0.601000048 \\
\hline & & Continued & on next page
\end{tabular}


Table S2 - Continued from previous page

\begin{tabular}{llll}
\hline \hline $\mathrm{N}$ & -5.623000432 & 1.880001393 & 0.462000033 \\
$\mathrm{~N}$ & -3.002000229 & 4.323999580 & 0.297001022 \\
$\mathrm{~N}$ & -5.601000428 & -0.414998780 & 0.314001026 \\
$\mathrm{O}$ & -1.508000116 & 1.646000377 & 0.000000000 \\
$\mathrm{C}$ & -4.225000322 & -1.638000874 & -2.969000227 \\
$\mathrm{C}$ & -4.816000370 & -2.892999971 & -3.095999238 \\
$\mathrm{C}$ & -2.817000217 & -1.569999872 & -2.861000220 \\
$\mathrm{C}$ & -2.912000223 & -4.030000056 & -2.958000225 \\
$\mathrm{C}$ & -6.319000481 & -1.271999848 & -3.215000247 \\
$\mathrm{H}$ & -1.210000092 & -2.859999970 & -2.910999221 \\
$\mathrm{H}$ & -6.879602958 & -3.337603953 & -3.366834387 \\
$\mathrm{H}$ & -7.270000557 & -0.772998810 & -3.330000253 \\
$\mathrm{H}$ & -1.171000089 & -5.086000139 & -2.814998213 \\
$\mathrm{H}$ & -2.645000201 & -6.054000214 & -2.927000223 \\
$\mathrm{~N}$ & -2.229000170 & -2.821999963 & -2.910000224 \\
$\mathrm{~N}$ & -6.159000469 & -2.653999950 & -3.250999249 \\
$\mathrm{~N}$ & -4.236000324 & -4.099000065 & -3.070999232 \\
$\mathrm{~N}$ & -5.175000396 & -0.640998799 & -3.024000233 \\
$\mathrm{~N}$ & -2.186000169 & -5.143000143 & -2.894998224 \\
$\mathrm{O}$ & -2.124000164 & -0.534999792 & -2.736000209 \\
$\mathrm{C}$ & -4.242000326 & -1.546998871 & 6.911001526 \\
$\mathrm{C}$ & -4.887000372 & -2.749999958 & 7.128001542 \\
$\mathrm{C}$ & -2.847000217 & -1.541000869 & 6.657000510 \\
$\mathrm{C}$ & -3.034000233 & -3.994000054 & 6.977000533 \\
$\mathrm{C}$ & -6.306000479 & -1.080999834 & 7.213000553 \\
$\mathrm{H}$ & -1.312000102 & -2.920999972 & 6.595000506 \\
$\mathrm{H}$ & -6.948364239 & -3.098049704 & 7.486294704 \\
$\mathrm{H}$ & -7.233000553 & -0.541000794 & 7.338000559 \\
$\mathrm{H}$ & -1.328000099 & -5.105000142 & 6.916003526 \\
$\mathrm{H}$ & -2.818000214 & -6.005000211 & 7.220000552 \\
$\mathrm{~N}$ & -2.318000179 & -2.825999966 & 6.732001516 \\
$\mathrm{~N}$ & -6.205000471 & -2.451999940 & 7.313002559 \\
$\mathrm{~N}$ & -4.343000334 & -3.992000055 & 7.178999549 \\
$\mathrm{~N}$ & -5.160000396 & -0.499999786 & 6.951002531 \\
$\mathrm{~N}$ & -2.340000178 & -5.121000139 & 7.043001536 \\
$\mathrm{O}$ & -2.137000165 & -0.572000796 & 6.409003487 \\
$\mathrm{C}$ & -4.561000347 & 0.509000287 & 3.629999276 \\
$\mathrm{C}$ & -5.646000433 & -0.340999777 & 3.546000272 \\
$\mathrm{C}$ & -3.258000248 & -0.037999753 & 3.636999280 \\
& -6.427000338 & -2.205999920 & 3.583002272 \\
& & 1.771000383 & 3.562002269 \\
\hline
\end{tabular}


Table S2 - Continued from previous page

\begin{tabular}{cccc}
\hline \hline $\mathrm{H}$ & -2.388000185 & -1.916999894 & 3.496001267 \\
$\mathrm{H}$ & -7.691312306 & 0.182951995 & 3.376259298 \\
$\mathrm{H}$ & -6.922000528 & 2.638000451 & 3.562002269 \\
$\mathrm{H}$ & -3.324000256 & -3.908000046 & 3.731001284 \\
$\mathrm{H}$ & -5.076000387 & -4.130000067 & 3.692999283 \\
$\mathrm{~N}$ & -3.283000248 & -1.433000862 & 3.566000272 \\
$\mathrm{~N}$ & -6.739000515 & 0.472000288 & 3.474001268 \\
$\mathrm{~N}$ & -5.650000431 & -1.683999881 & 3.539999270 \\
$\mathrm{~N}$ & -4.972000378 & 1.833000388 & 3.646002278 \\
$\mathrm{~N}$ & -4.263000323 & -3.514000019 & 3.676001278 \\
$\mathrm{O}$ & -2.166000163 & 0.566999293 & 3.695002282 \\
$\mathrm{Sr}$ & 0.000000021 & 0.000000259 & -1.341999102 \\
$\mathrm{Sr}$ & 0.000000000 & 0.000000000 & 1.847501141 \\
\hline
\end{tabular}


Table S3: The Cartesian coordinates of the structure $\mathbf{3}$ in $\AA$.

\begin{tabular}{cccc}
\hline \hline $\mathrm{C}$ & -3.968293845 & -1.889096462 & 2.083121158 \\
$\mathrm{C}$ & -4.431291881 & -3.178094563 & 2.083121158 \\
$\mathrm{C}$ & -2.564290738 & -1.687097447 & 2.043124158 \\
$\mathrm{C}$ & -2.438291725 & -4.148095638 & 2.011123154 \\
$\mathrm{C}$ & -6.096293007 & -1.730096453 & 2.151122165 \\
$\mathrm{H}$ & -0.881292605 & -2.820096534 & 1.975123151 \\
$\mathrm{H}$ & -6.459977221 & -3.825142954 & 2.141483503 \\
$\mathrm{H}$ & -7.114294084 & -1.373094425 & 2.185122168 \\
$\mathrm{H}$ & -0.587293585 & -5.148095713 & 1.932124145 \\
$\mathrm{H}$ & -2.013292696 & -6.105096786 & 1.969123149 \\
$\mathrm{~N}$ & -1.879293681 & -2.882096538 & 2.007125151 \\
$\mathrm{~N}$ & -5.803292983 & -3.071095553 & 2.128123164 \\
$\mathrm{~N}$ & -3.747293826 & -4.324094651 & 2.049125155 \\
$\mathrm{~N}$ & -5.021291921 & -0.987096395 & 2.126124160 \\
$\mathrm{~N}$ & -1.596292663 & -5.185094717 & 1.974124150 \\
$\mathrm{O}$ & -1.955293689 & -0.612096366 & 2.035125157 \\
$\mathrm{C}$ & -1.945291689 & -3.866094616 & 5.358123411 \\
$\mathrm{C}$ & -1.596292663 & -5.179093715 & 5.535125422 \\
$\mathrm{C}$ & -0.917291608 & -2.935094545 & 5.071124389 \\
$\mathrm{C}$ & 0.565706503 & -4.876096691 & 5.209125397 \\
$\mathrm{C}$ & -3.784291830 & -4.872094693 & 5.762122438 \\
$\mathrm{H}$ & 1.109709547 & -2.951096547 & 4.814124368 \\
$\mathrm{H}$ & -2.900513801 & -6.793998266 & 5.971783094 \\
$\mathrm{H}$ & -4.818292909 & -5.131097714 & 5.936122453 \\
$\mathrm{H}$ & 2.639707661 & -4.686093679 & 4.901122372 \\
$\mathrm{H}$ & 2.022707616 & -6.251096800 & 5.246123401 \\
$\mathrm{~N}$ & 0.323705486 & -3.536096588 & 5.014125385 \\
$\mathrm{~N}$ & -2.783292753 & -5.817094762 & 5.793124440 \\
$\mathrm{~N}$ & -0.378293570 & -5.770097758 & 5.479121419 \\
$\mathrm{~N}$ & -3.321290795 & -3.667096601 & 5.500121421 \\
$\mathrm{~N}$ & 1.840708601 & -5.266095720 & 5.112124392 \\
$\mathrm{O}$ & -1.059293618 & -1.715096453 & 4.884124373 \\
$\mathrm{C}$ & 0.179708472 & -4.225095643 & 8.562121656 \\
$\mathrm{C}$ & 1.110708543 & -5.211095719 & 8.780124669 \\
$\mathrm{C}$ & 0.626707505 & -2.909097543 & 8.272124633 \\
$\mathrm{C}$ & 2.852709680 & -3.901093616 & 8.491122648 \\
$\mathrm{C}$ & -0.911290611 & -6.010095780 & 8.957122685 \\
$\mathrm{H}$ & 2.446707649 & -1.955094470 & 8.060122617 \\
$\mathrm{H}$ & 0.797012191 & -7.273398055 & 9.235823985 \\
$\mathrm{H}$ & -1.683294668 & -6.748094833 & 9.119124695 \\
\hline & & Continued & on $n$ ext page
\end{tabular}


Table S3 - Continued from previous page

\begin{tabular}{cccc}
\hline \hline $\mathrm{H}$ & 4.601705809 & -2.756097530 & 8.225123628 \\
$\mathrm{H}$ & 4.787706823 & -4.422093658 & 8.597121656 \\
$\mathrm{~N}$ & 2.020708612 & -2.838094535 & 8.256122631 \\
$\mathrm{~N}$ & 0.419706494 & -6.369096807 & 9.036124689 \\
$\mathrm{~N}$ & 2.461706649 & -5.133096713 & 8.763122670 \\
$\mathrm{~N}$ & -1.100293626 & -4.748094684 & 8.678123664 \\
$\mathrm{~N}$ & 4.165708779 & -3.642095596 & 8.433124642 \\
$\mathrm{O}$ & -0.038293541 & -1.897096464 & 8.048121612 \\
$\mathrm{C}$ & -1.863294685 & 4.116903994 & 1.598124124 \\
$\mathrm{C}$ & -3.141294778 & 4.642905037 & 1.545123117 \\
$\mathrm{C}$ & -1.677293671 & 2.714903886 & 1.749122132 \\
$\mathrm{C}$ & -4.089292853 & 2.613905878 & 1.768123135 \\
$\mathrm{C}$ & -1.643293667 & 6.237906157 & 1.367122104 \\
$\mathrm{H}$ & -2.848291759 & 0.994905754 & 1.937121150 \\
$\mathrm{H}$ & -3.731088254 & 6.671660879 & 1.327314841 \\
$\mathrm{H}$ & -1.252294637 & 7.237906232 & 1.255122094 \\
$\mathrm{H}$ & -5.070291925 & 0.756903736 & 1.960124152 \\
$\mathrm{H}$ & -6.043291000 & 2.163904844 & 1.814124138 \\
$\mathrm{~N}$ & -2.856294761 & 1.989904834 & 1.831122142 \\
$\mathrm{~N}$ & -2.998292771 & 5.994906139 & 1.398124107 \\
$\mathrm{~N}$ & -4.269292865 & 3.910906981 & 1.627124122 \\
$\mathrm{~N}$ & -0.935292609 & 5.145904072 & 1.484122115 \\
$\mathrm{~N}$ & -5.119291928 & 1.761904816 & 1.863124141 \\
$\mathrm{O}$ & -0.604291589 & 2.089903840 & 1.814124138 \\
$\mathrm{C}$ & -3.647290820 & 2.382902864 & 5.171123395 \\
$\mathrm{C}$ & -4.975293919 & 2.067904836 & 5.338122406 \\
$\mathrm{C}$ & -2.694291744 & 1.328905781 & 5.074122385 \\
$\mathrm{C}$ & -4.651292893 & -0.133096328 & 5.336123407 \\
$\mathrm{C}$ & -4.648294897 & 4.249904006 & 5.261123401 \\
$\mathrm{H}$ & -2.704293745 & -0.715096373 & 5.111121389 \\
$\mathrm{H}$ & -6.603282823 & 3.425277119 & 5.513101631 \\
$\mathrm{H}$ & -4.909291916 & 5.296905086 & 5.274123402 \\
$\mathrm{H}$ & -4.362291872 & -2.227094492 & 5.328124406 \\
$\mathrm{H}$ & -5.966291996 & -1.644093445 & 5.514125420 \\
$\mathrm{~N}$ & -3.298291793 & 0.087904686 & 5.168124394 \\
$\mathrm{~N}$ & -5.621293968 & 3.276903931 & 5.396125413 \\
$\mathrm{~N}$ & -5.543290966 & 0.842905744 & 5.428123417 \\
$\mathrm{~N}$ & -3.445291804 & 3.759905967 & 5.123122394 \\
$\mathrm{~N}$ & -4.985292919 & -1.435094430 & 5.400123410 \\
$\mathrm{C}$ & -1.469293653 & 1.355906785 & 4.925124376 \\
& -4.304292871 & 0.451902712 & 8.573123653 \\
\hline & & Continued on next page
\end{tabular}


Table S3 - Continued from previous page

\begin{tabular}{cccc}
\hline \hline $\mathrm{C}$ & -5.289293945 & -0.490094355 & 8.813125670 \\
$\mathrm{C}$ & -2.983293765 & -0.030095321 & 8.399123643 \\
$\mathrm{C}$ & -3.950292844 & -2.272096492 & 8.746123665 \\
$\mathrm{C}$ & -6.132292010 & 1.564905800 & 8.777122668 \\
$\mathrm{H}$ & -1.990293690 & -1.824094462 & 8.385123640 \\
$\mathrm{H}$ & -7.365926285 & -0.139638123 & 9.123965889 \\
$\mathrm{H}$ & -6.872294067 & 2.348906861 & 8.829123672 \\
$\mathrm{H}$ & -2.790292752 & -4.032094630 & 8.694121666 \\
$\mathrm{H}$ & -4.475293879 & -4.193093638 & 8.986121688 \\
$\mathrm{~N}$ & -2.896291760 & -1.416097427 & 8.501124648 \\
$\mathrm{~N}$ & -6.455294034 & 0.231905696 & 8.943122681 \\
$\mathrm{~N}$ & -5.184293934 & -1.828095460 & 8.908122681 \\
$\mathrm{~N}$ & -4.854291911 & 1.727903812 & 8.556125654 \\
$\mathrm{~N}$ & -3.688291822 & -3.584096594 & 8.814124672 \\
$\mathrm{O}$ & -1.948293690 & 0.622904726 & 8.176124625 \\
$\mathrm{C}$ & 4.268705785 & 2.005903831 & 1.549125121 \\
$\mathrm{C}$ & 4.740705824 & 3.298903930 & 1.374122103 \\
$\mathrm{C}$ & 2.869707679 & 1.809904817 & 1.667125128 \\
$\mathrm{C}$ & 2.739706667 & 4.252906003 & 1.408122107 \\
$\mathrm{C}$ & 6.401708948 & 1.853903821 & 1.418124107 \\
$\mathrm{H}$ & 1.161707551 & 2.977903910 & 1.655124129 \\
$\mathrm{H}$ & 6.767140976 & 3.927387359 & 1.165022539 \\
$\mathrm{H}$ & 7.420707026 & 1.494905794 & 1.385123106 \\
$\mathrm{H}$ & 0.891706528 & 5.274905082 & 1.410125106 \\
$\mathrm{H}$ & 2.318708636 & 6.208903154 & 1.213122095 \\
$\mathrm{~N}$ & 2.157709623 & 3.012903910 & 1.584125121 \\
$\mathrm{~N}$ & 6.111707925 & 3.182905922 & 1.292125099 \\
$\mathrm{~N}$ & 4.031706769 & 4.439906018 & 1.298121101 \\
$\mathrm{~N}$ & 5.327707869 & 1.113905763 & 1.574122120 \\
$\mathrm{~N}$ & 1.898706607 & 5.300906084 & 1.350123105 \\
$\mathrm{O}$ & 2.275705635 & 0.730906734 & 1.828124141 \\
$\mathrm{C}$ & 2.405706646 & 4.165903997 & 4.800124364 \\
$\mathrm{C}$ & 2.087705622 & 5.501906098 & 4.864123373 \\
$\mathrm{C}$ & 1.360708566 & 3.207903927 & 4.748122365 \\
$\mathrm{C}$ & -0.104291549 & 5.159904075 & 4.838122371 \\
$\mathrm{C}$ & 4.264707788 & 5.206904080 & 4.859122373 \\
$\mathrm{H}$ & -0.699292595 & 3.213903924 & 4.735122364 \\
$\mathrm{H}$ & 3.430722094 & 7.162203469 & 4.953265958 \\
$\mathrm{H}$ & 5.308706866 & 5.484904099 & 4.875121370 \\
$\mathrm{H}$ & -2.210290706 & 5.061904069 & 4.810122370 \\
& -1.483293656 & 6.615905187 & 4.895122375 \\
\hline & & Continued on next page
\end{tabular}


Table S3 - Continued from previous page

\begin{tabular}{cccc}
\hline \hline $\mathrm{N}$ & 0.106706470 & 3.804903972 & 4.771125367 \\
$\mathrm{~N}$ & 3.293707711 & 6.172904151 & 4.903125377 \\
$\mathrm{~N}$ & 0.862707525 & 6.071906144 & 4.887122374 \\
$\mathrm{~N}$ & 3.794706748 & 3.984903984 & 4.797122368 \\
$\mathrm{~N}$ & -1.365293643 & 5.613906108 & 4.854121373 \\
$\mathrm{O}$ & 1.491709574 & 1.963903832 & 4.688125360 \\
$\mathrm{C}$ & 0.316705482 & 4.960906061 & 8.125121622 \\
$\mathrm{C}$ & -0.606294588 & 5.966907138 & 8.299125632 \\
$\mathrm{C}$ & -0.126291548 & 3.606905954 & 8.036124614 \\
$\mathrm{C}$ & -2.361291720 & 4.618903033 & 8.324122637 \\
$\mathrm{C}$ & 1.434706569 & 6.756904195 & 8.197124627 \\
$\mathrm{H}$ & -1.920293689 & 2.636904879 & 8.099124620 \\
$\mathrm{H}$ & -0.240718566 & 8.061066076 & 8.465645086 \\
$\mathrm{H}$ & 2.214708627 & 7.503905252 & 8.198123624 \\
$\mathrm{H}$ & -4.149293859 & 3.491903948 & 8.361121636 \\
$\mathrm{H}$ & -4.276292864 & 5.195907078 & 8.535125652 \\
$\mathrm{~N}$ & -1.510293655 & 3.547903950 & 8.150123623 \\
$\mathrm{~N}$ & 0.114706471 & 7.133906223 & 8.347121638 \\
$\mathrm{~N}$ & -1.955293689 & 5.884906128 & 8.407123644 \\
$\mathrm{~N}$ & 1.604707581 & 5.479903098 & 8.063125618 \\
$\mathrm{~N}$ & -3.684293819 & 4.386905017 & 8.416121642 \\
$\mathrm{O}$ & 0.581709505 & 2.602903881 & 7.882125604 \\
$\mathrm{C}$ & 2.159708627 & -4.114095634 & 1.864123143 \\
$\mathrm{C}$ & 3.443708722 & -4.580094671 & 1.767123133 \\
$\mathrm{C}$ & 1.917707605 & -2.709096526 & 1.902122145 \\
$\mathrm{C}$ & 4.358705791 & -2.560094516 & 1.733123130 \\
$\mathrm{C}$ & 2.022707616 & -6.241094794 & 1.841125142 \\
$\mathrm{H}$ & 3.060709693 & -0.985097396 & 1.850123140 \\
$\mathrm{H}$ & 4.121596002 & -6.604774976 & 1.692005798 \\
$\mathrm{H}$ & 1.686708591 & -7.268094874 & 1.847121139 \\
$\mathrm{H}$ & 5.426706873 & -0.734094376 & 1.676124125 \\
$\mathrm{H}$ & 6.333708941 & -2.189095485 & 1.592124122 \\
$\mathrm{~N}$ & 3.106706695 & -1.983094471 & 1.830123140 \\
$\mathrm{~N}$ & 3.364706719 & -5.954095773 & 1.753123135 \\
$\mathrm{~N}$ & 4.590708813 & -3.870095614 & 1.697124128 \\
$\mathrm{~N}$ & 1.254705557 & -5.174096715 & 1.911124148 \\
$\mathrm{~N}$ & 5.430708876 & -1.743096454 & 1.671123125 \\
$\mathrm{O}$ & 0.816705522 & -2.136094484 & 1.988124153 \\
$\mathrm{C}$ & 4.157709778 & -2.076096478 & 5.116122389 \\
$\mathrm{C}$ & 5.502706881 & -1.765096453 & 5.217124398 \\
$\mathrm{C}$ & 3.217706708 & -1.038094397 & 4.917125375 \\
\hline & & Continued & on next page
\end{tabular}


Table S3 - Continued from previous page

\begin{tabular}{cccc}
\hline \hline $\mathrm{C}$ & 5.181707856 & 0.423903711 & 4.961123378 \\
$\mathrm{C}$ & 5.155706853 & -3.948094621 & 5.406123412 \\
$\mathrm{H}$ & 3.240705709 & 1.024904760 & 4.711123361 \\
$\mathrm{H}$ & 7.108760482 & -3.117260055 & 5.514083932 \\
$\mathrm{H}$ & 5.389707874 & -4.995095700 & 5.535125422 \\
$\mathrm{H}$ & 5.005708842 & 2.516905874 & 4.719123362 \\
$\mathrm{H}$ & 6.580705962 & 1.870905825 & 4.942122380 \\
$\mathrm{~N}$ & 3.816705752 & 0.219904697 & 4.848124371 \\
$\mathrm{~N}$ & 6.125707928 & -2.972096549 & 5.402122414 \\
$\mathrm{~N}$ & 6.066705924 & -0.550096361 & 5.148124394 \\
$\mathrm{~N}$ & 3.969705764 & -3.445096585 & 5.237124398 \\
$\mathrm{~N}$ & 5.586706884 & 1.705904808 & 4.870124370 \\
$\mathrm{O}$ & 1.983705613 & -1.155095407 & 4.808124366 \\
$\mathrm{C}$ & 4.816705827 & 0.238905700 & 8.303123635 \\
$\mathrm{C}$ & 5.820707905 & 1.173903769 & 8.356124637 \\
$\mathrm{C}$ & 3.480707727 & 0.686903730 & 8.115123617 \\
$\mathrm{C}$ & 4.503706803 & 2.914904903 & 8.081122619 \\
$\mathrm{C}$ & 6.603708964 & -0.874097388 & 8.590121657 \\
$\mathrm{H}$ & 2.523706653 & 2.479902869 & 7.879123603 \\
$\mathrm{H}$ & 7.902477624 & 0.810237574 & 8.628164421 \\
$\mathrm{H}$ & 7.335707020 & -1.657094446 & 8.728122664 \\
$\mathrm{H}$ & 3.338709717 & 4.645904032 & 7.813125596 \\
$\mathrm{H}$ & 5.034708845 & 4.843905050 & 8.001124614 \\
$\mathrm{~N}$ & 3.424707719 & 2.067904836 & 8.013122612 \\
$\mathrm{~N}$ & 6.974706994 & 0.446905713 & 8.543124653 \\
$\mathrm{~N}$ & 5.755708899 & 2.513903872 & 8.255122629 \\
$\mathrm{~N}$ & 5.319708868 & -1.042096400 & 8.451121648 \\
$\mathrm{~N}$ & 4.241709781 & 4.218905004 & 7.960124606 \\
$\mathrm{O}$ & 2.461706649 & -0.010095321 & 8.045123617 \\
$\mathrm{Na}$ & 0.332708485 & 0.266905701 & 7.012122535 \\
$\mathrm{Na}$ & 0.121706470 & 0.097902686 & 3.532123271 \\
\hline & & &
\end{tabular}


Table S4: The Cartesian coordinates of the structure 4 in $\AA$.

\begin{tabular}{cccc}
\hline \hline $\mathrm{C}$ & -4.340291873 & -1.779096456 & -1.429875107 \\
$\mathrm{C}$ & -5.606294968 & -1.268094419 & -1.568875122 \\
$\mathrm{C}$ & -3.256292789 & -0.860097384 & -1.333879100 \\
$\mathrm{C}$ & -4.970292919 & 0.870905745 & -1.538877116 \\
$\mathrm{C}$ & -5.629293969 & -3.461095582 & -1.534879119 \\
$\mathrm{H}$ & -2.964292767 & 1.193903769 & -1.339875101 \\
$\mathrm{H}$ & -7.438755600 & -2.346770578 & -1.743547244 \\
$\mathrm{H}$ & -6.057291004 & -4.453095661 & -1.559876118 \\
$\mathrm{H}$ & -4.616292893 & 2.955904906 & -1.524877118 \\
$\mathrm{H}$ & -6.241293019 & 2.423903866 & -1.685876127 \\
$\mathrm{~N}$ & -3.657293820 & 0.475904716 & -1.397878108 \\
$\mathrm{~N}$ & -6.444292032 & -2.356095501 & -1.638879123 \\
$\mathrm{~N}$ & -5.988294995 & 0.029905685 & -1.629876124 \\
$\mathrm{~N}$ & -4.358293874 & -3.175096562 & -1.407876108 \\
$\mathrm{~N}$ & -5.265290942 & 2.184904846 & -1.590878121 \\
$\mathrm{O}$ & -2.057290699 & -1.157094406 & -1.204877090 \\
$\mathrm{C}$ & -4.701291898 & 0.566904724 & -4.884877372 \\
$\mathrm{C}$ & -5.531293962 & 1.646906805 & -5.068875386 \\
$\mathrm{C}$ & -3.300290792 & 0.775904739 & -4.800878368 \\
$\mathrm{C}$ & -3.915292839 & 3.133905919 & -5.109876389 \\
$\mathrm{C}$ & -6.691294053 & -0.200093337 & -4.949876377 \\
$\mathrm{H}$ & -2.031293697 & 2.392904864 & -4.891877376 \\
$\mathrm{H}$ & -7.662934154 & 1.677683870 & -5.236857042 \\
$\mathrm{H}$ & -7.568293116 & -0.830094384 & -4.941876376 \\
$\mathrm{H}$ & -2.494291732 & 4.701903040 & -5.168878392 \\
$\mathrm{H}$ & -4.150293856 & 5.120906072 & -5.340878408 \\
$\mathrm{~N}$ & -2.996293767 & 2.131903844 & -4.929875377 \\
$\mathrm{~N}$ & -6.818293058 & 1.156904770 & -5.112878390 \\
$\mathrm{~N}$ & -5.231294939 & 2.961905908 & -5.192876395 \\
$\mathrm{~N}$ & -5.456292956 & -0.603093367 & -4.811875370 \\
$\mathrm{~N}$ & -3.456292806 & 4.398906016 & -5.217877401 \\
$\mathrm{O}$ & -2.384294722 & -0.063096322 & -4.637876355 \\
$\mathrm{C}$ & -3.996293847 & 2.490904871 & -8.336877635 \\
$\mathrm{C}$ & -4.170293856 & 3.849905973 & -8.490876649 \\
$\mathrm{C}$ & -2.663293742 & 2.011904833 & -8.238877628 \\
$\mathrm{C}$ & -2.022290694 & 4.386905017 & -8.473878645 \\
$\mathrm{C}$ & -6.105292005 & 2.812902893 & -8.443875645 \\
$\mathrm{H}$ & -0.750291597 & 2.797903893 & -8.257878631 \\
$\mathrm{H}$ & -6.002023880 & 4.924930946 & -8.672144723 \\
$\mathrm{H}$ & -7.177294090 & 2.685904883 & -8.464875647 \\
\hline & & Continued \\
& on next page
\end{tabular}


Table S4 - Continued from previous page

\begin{tabular}{ccrc}
\hline \hline $\mathrm{H}$ & -0.006292542 & 5.014903064 & -8.461877646 \\
$\mathrm{H}$ & -1.217290630 & 6.219905156 & -8.636876658 \\
$\mathrm{~N}$ & -1.717290671 & 3.046904914 & -8.317876637 \\
$\mathrm{~N}$ & -5.529290962 & 4.050905992 & -8.559876653 \\
$\mathrm{~N}$ & -3.260293787 & 4.838904050 & -8.566876652 \\
$\mathrm{~N}$ & -5.231294939 & 1.868902820 & -8.310876632 \\
$\mathrm{~N}$ & -0.988294616 & 5.242904082 & -8.531877652 \\
$\mathrm{O}$ & -2.264291715 & 0.833902745 & -8.095876620 \\
$\mathrm{C}$ & 1.612707582 & -4.398095655 & -1.635877126 \\
$\mathrm{C}$ & 1.089708541 & -5.676095754 & -1.724877130 \\
$\mathrm{C}$ & 0.730707514 & -3.297093572 & -1.445878110 \\
$\mathrm{C}$ & -1.012292619 & -5.013097702 & -1.477876114 \\
$\mathrm{C}$ & 3.289709713 & -5.722096757 & -1.915875144 \\
$\mathrm{H}$ & -1.313291639 & -3.002095549 & -1.244878096 \\
$\mathrm{H}$ & 2.134787712 & -7.506534183 & -2.003850332 \\
$\mathrm{H}$ & 4.284708788 & -6.125096786 & -2.038877156 \\
$\mathrm{H}$ & -3.069291773 & -4.581094668 & -1.245877098 \\
$\mathrm{H}$ & -2.619291739 & -6.225095798 & -1.457875113 \\
$\mathrm{~N}$ & -0.604291589 & -3.694097600 & -1.375878104 \\
$\mathrm{~N}$ & 2.164709627 & -6.512094819 & -1.902879143 \\
$\mathrm{~N}$ & -0.201291554 & -6.046094783 & -1.653878128 \\
$\mathrm{~N}$ & 2.987707686 & -4.471093662 & -1.760877133 \\
$\mathrm{~N}$ & -2.334291716 & -5.258096724 & -1.390878104 \\
$\mathrm{O}$ & 0.957708531 & -2.080094481 & -1.339875101 \\
$\mathrm{C}$ & -1.029290618 & -4.767095682 & -4.777879367 \\
$\mathrm{C}$ & -2.141294703 & -5.591096748 & -4.850877368 \\
$\mathrm{C}$ & -1.210290631 & -3.377095578 & -4.642877355 \\
$\mathrm{C}$ & -3.599294814 & -3.920094619 & -4.680879357 \\
$\mathrm{C}$ & -0.274293561 & -6.777097837 & -4.974877377 \\
$\mathrm{H}$ & -2.790292752 & -2.058095477 & -4.505876345 \\
$\mathrm{H}$ & -2.193736058 & -7.712405887 & -5.056860226 \\
$\mathrm{H}$ & 0.369707489 & -7.641095903 & -5.057878384 \\
$\mathrm{H}$ & -5.068292926 & -2.421094507 & -4.516877347 \\
$\mathrm{H}$ & -5.598291966 & -4.047093630 & -4.672875355 \\
$\mathrm{~N}$ & -2.559293733 & -3.026093552 & -4.600877351 \\
$\mathrm{~N}$ & -1.654290664 & -6.874097847 & -4.977876378 \\
$\mathrm{~N}$ & -3.428292800 & -5.232095722 & -4.807878367 \\
$\mathrm{~N}$ & 0.118707469 & -5.530095740 & -4.857877372 \\
$\mathrm{~N}$ & -4.828290908 & -3.396096582 & -4.623876352 \\
$\mathrm{O}$ & -0.372293568 & -2.457093509 & -4.559876348 \\
$\mathrm{C}$ & -3.338292794 & -3.934094623 & -7.978875610 \\
\hline & & Continued on next page
\end{tabular}


Table S4 - Continued from previous page

\begin{tabular}{cccc}
\hline \hline $\mathrm{C}$ & -4.700292902 & -4.085095631 & -8.046876617 \\
$\mathrm{C}$ & -2.791291754 & -2.627095521 & -7.906877606 \\
$\mathrm{C}$ & -5.130292931 & -1.918096466 & -7.988877610 \\
$\mathrm{C}$ & -3.664293819 & -6.039094784 & -8.069879619 \\
$\mathrm{H}$ & -3.509294808 & -0.681096369 & -7.872876602 \\
$\mathrm{H}$ & -5.791585895 & -5.914251652 & -8.163522692 \\
$\mathrm{H}$ & -3.540292810 & -7.112096865 & -8.099877619 \\
$\mathrm{H}$ & -5.717290975 & 0.115904687 & -7.931878606 \\
$\mathrm{H}$ & -6.957293073 & -1.068093402 & -8.032876614 \\
$\mathrm{~N}$ & -3.781293829 & -1.642095446 & -7.917878603 \\
$\mathrm{~N}$ & -4.909291916 & -5.447095734 & -8.105878621 \\
$\mathrm{~N}$ & -5.658292972 & -3.129094560 & -8.056878617 \\
$\mathrm{~N}$ & -2.706292749 & -5.160096712 & -7.994878613 \\
$\mathrm{~N}$ & -5.970292994 & -0.860097384 & -7.989877613 \\
$\mathrm{O}$ & -1.602292665 & -2.291097496 & -7.839875601 \\
$\mathrm{C}$ & 4.174707777 & 1.500902796 & -1.870877144 \\
$\mathrm{C}$ & 5.431707873 & 0.960905751 & -2.060876155 \\
$\mathrm{C}$ & 3.098707699 & 0.619905726 & -1.600877121 \\
$\mathrm{C}$ & 4.778707826 & -1.152097406 & -1.770879133 \\
$\mathrm{C}$ & 5.461706878 & 3.152903922 & -2.242875171 \\
$\mathrm{H}$ & 2.798708676 & -1.404096428 & -1.379876107 \\
$\mathrm{H}$ & 7.246216485 & 2.015866185 & -2.480004317 \\
$\mathrm{H}$ & 5.880708911 & 4.135904997 & -2.398877185 \\
$\mathrm{H}$ & 4.242708783 & -3.179093565 & -1.494878113 \\
$\mathrm{H}$ & 5.889707909 & -2.828095535 & -1.832875142 \\
$\mathrm{~N}$ & 3.496706729 & -0.713094374 & -1.567876120 \\
$\mathrm{~N}$ & 6.262708939 & 2.040903836 & -2.300878178 \\
$\mathrm{~N}$ & 5.790708900 & -0.331094346 & -2.022878154 \\
$\mathrm{~N}$ & 4.207708783 & 2.882902899 & -1.987878154 \\
$\mathrm{~N}$ & 4.949709840 & -2.485097511 & -1.694879130 \\
$\mathrm{O}$ & 1.916708608 & 0.869905748 & -1.402879108 \\
$\mathrm{C}$ & 4.384706794 & -1.176095409 & -5.131879393 \\
$\mathrm{C}$ & 5.158708855 & -2.307095498 & -5.295877403 \\
$\mathrm{C}$ & 2.991709689 & -1.288094419 & -4.918878375 \\
$\mathrm{C}$ & 3.445707721 & -3.696095604 & -5.082875389 \\
$\mathrm{C}$ & 6.374708949 & -0.492097360 & -5.415875414 \\
$\mathrm{H}$ & 1.626707585 & -2.827096538 & -4.765878362 \\
$\mathrm{H}$ & 7.259598827 & -2.410790505 & -5.628058250 \\
$\mathrm{H}$ & 7.272708013 & 0.096903689 & -5.530877420 \\
$\mathrm{H}$ & 1.847708600 & -5.069097709 & -4.873876374 \\
\hline & 3.417707720 & -5.710095757 & -5.146878393 \\
\hline & & & \\
& & & \\
& &
\end{tabular}


Table S4 - Continued from previous page

\begin{tabular}{cccc}
\hline \hline $\mathrm{N}$ & 2.595709658 & -2.626096519 & -4.908875375 \\
$\mathrm{~N}$ & 6.441705953 & -1.855096459 & -5.478875420 \\
$\mathrm{~N}$ & 4.748708820 & -3.595094596 & -5.282876401 \\
$\mathrm{~N}$ & 5.179708857 & -0.030095321 & -5.211877399 \\
$\mathrm{~N}$ & 2.829706678 & -4.896096697 & -5.032876384 \\
$\mathrm{O}$ & 2.140707623 & -0.397096349 & -4.751878365 \\
$\mathrm{C}$ & 3.127706697 & -3.278096569 & -8.402879643 \\
$\mathrm{C}$ & 3.275705709 & -4.647096676 & -8.549878653 \\
$\mathrm{C}$ & 1.832708600 & -2.768094529 & -8.155877621 \\
$\mathrm{C}$ & 1.133707544 & -5.123094712 & -8.261876629 \\
$\mathrm{C}$ & 5.200708859 & -3.590096597 & -8.750878667 \\
$\mathrm{H}$ & -0.070291545 & -3.511094587 & -7.929875607 \\
$\mathrm{H}$ & 5.078730360 & -5.712727266 & -8.928525062 \\
$\mathrm{H}$ & 6.263708941 & -3.472096584 & -8.900876678 \\
$\mathrm{H}$ & -0.875291608 & -5.700093757 & -7.992875608 \\
$\mathrm{H}$ & 0.278707481 & -6.938096851 & -8.285878632 \\
$\mathrm{~N}$ & 0.876707528 & -3.780095608 & -8.099877619 \\
$\mathrm{~N}$ & 4.616709810 & -4.839093692 & -8.775876672 \\
$\mathrm{~N}$ & 2.338709637 & -5.613095747 & -8.493878651 \\
$\mathrm{~N}$ & 4.343706791 & -2.628095523 & -8.530877650 \\
$\mathrm{~N}$ & 0.084707466 & -5.952096774 & -8.175878627 \\
$\mathrm{O}$ & 1.503706573 & -1.583096443 & -7.996877612 \\
$\mathrm{C}$ & -1.650293666 & 4.078905993 & -1.868878145 \\
$\mathrm{C}$ & -1.165292631 & 5.354903087 & -2.104878159 \\
$\mathrm{C}$ & -0.755292597 & 2.994905909 & -1.640878127 \\
$\mathrm{C}$ & 0.945707532 & 4.696906040 & -1.932878149 \\
$\mathrm{C}$ & -3.344293796 & 5.334907088 & -2.147878166 \\
$\mathrm{H}$ & 1.309706558 & 2.721903890 & -1.546876117 \\
$\mathrm{H}$ & -2.271806635 & 7.149628074 & -2.476056258 \\
$\mathrm{H}$ & -4.344293871 & 5.729903116 & -2.244878171 \\
$\mathrm{H}$ & 3.008707688 & 4.255904003 & -1.792878137 \\
$\mathrm{H}$ & 2.561708655 & 5.881904132 & -2.117875160 \\
$\mathrm{~N}$ & 0.576708505 & 3.387903939 & -1.688878128 \\
$\mathrm{~N}$ & -2.264291715 & 6.167907152 & -2.285878173 \\
$\mathrm{~N}$ & 0.118707469 & 5.715903118 & -2.147878166 \\
$\mathrm{~N}$ & -3.035291770 & 4.105906992 & -1.902879143 \\
$\mathrm{~N}$ & 2.271707632 & 4.928905056 & -1.949876147 \\
$\mathrm{O}$ & -1.057290619 & 1.808905821 & -1.417878108 \\
$\mathrm{C}$ & 0.720709515 & 4.246905006 & -5.233876398 \\
$\mathrm{C}$ & 1.822706599 & 5.038905062 & -5.454877417 \\
& 0.872705524 & 2.852903899 & -5.008878381 \\
\hline & & Continued \\
\hline
\end{tabular}


Table S4 - Continued from previous page

\begin{tabular}{cccc}
\hline \hline $\mathrm{C}$ & 3.259706707 & 3.353903935 & -5.281877405 \\
$\mathrm{C}$ & -0.037294545 & 6.220904153 & -5.511876422 \\
$\mathrm{H}$ & 2.432707645 & 1.519903794 & -4.905877374 \\
$\mathrm{H}$ & 1.862791162 & 7.142476164 & -5.815758505 \\
$\mathrm{H}$ & -0.672292590 & 7.087905221 & -5.609876429 \\
$\mathrm{H}$ & 4.692709818 & 1.825903819 & -5.121877393 \\
$\mathrm{H}$ & 5.250707859 & 3.418905941 & -5.438878415 \\
$\mathrm{~N}$ & 2.208708630 & 2.483904872 & -5.051877387 \\
$\mathrm{~N}$ & 1.331709563 & 6.314906162 & -5.633878432 \\
$\mathrm{~N}$ & 3.118707699 & 4.657905037 & -5.491876422 \\
$\mathrm{~N}$ & -0.438291575 & 4.992904060 & -5.269876400 \\
$\mathrm{~N}$ & 4.469705799 & 2.798903895 & -5.281877405 \\
$\mathrm{O}$ & -0.057290544 & 2.042906836 & -4.798879364 \\
$\mathrm{C}$ & 2.498708653 & 3.123903918 & -8.493878651 \\
$\mathrm{C}$ & 3.836706752 & 3.291903931 & -8.720876667 \\
$\mathrm{C}$ & 1.975706612 & 1.807905818 & -8.275876632 \\
$\mathrm{C}$ & 4.313707791 & 1.123903763 & -8.571877652 \\
$\mathrm{C}$ & 2.767706674 & 5.237906083 & -8.754876670 \\
$\mathrm{H}$ & 2.733705670 & -0.111096329 & -8.191876623 \\
$\mathrm{H}$ & 4.870569504 & 5.127081322 & -9.074932722 \\
$\mathrm{H}$ & 2.616709660 & 6.303905160 & -8.838876674 \\
$\mathrm{H}$ & 4.978708838 & -0.876096386 & -8.454877647 \\
$\mathrm{H}$ & 6.151708930 & 0.336905707 & -8.769876670 \\
$\mathrm{~N}$ & 2.985708687 & 0.846903747 & -8.333879634 \\
$\mathrm{~N}$ & 4.008707768 & 4.654906036 & -8.889878676 \\
$\mathrm{~N}$ & 4.794706828 & 2.346903862 & -8.773877668 \\
$\mathrm{~N}$ & 1.840708601 & 4.345904014 & -8.517877649 \\
$\mathrm{~N}$ & 5.183706854 & 0.101904689 & -8.601876657 \\
$\mathrm{O}$ & 0.777708519 & 1.533903797 & -8.059876618 \\
$\mathrm{Ca}$ & -0.079293544 & -0.215096338 & -3.252877246 \\
$\mathrm{Ca}$ & -0.308294564 & -0.309094341 & -6.835877523 \\
\hline & & & \\
\hline
\end{tabular}


Table S5: The Cartesian coordinates of the structure $\mathbf{5}$ in $\AA$.

\begin{tabular}{cccc}
\hline \hline $\mathrm{C}$ & 0.006000002 & 14.537001108 & 9.192000700 \\
$\mathrm{C}$ & 0.300000023 & 23.541001795 & 12.760000977 \\
$\mathrm{C}$ & 0.444000032 & 22.199000693 & 9.745000742 \\
$\mathrm{C}$ & 0.639000049 & 22.199000693 & 13.028000995 \\
$\mathrm{C}$ & 0.676000054 & 12.586000962 & 12.427000947 \\
$\mathrm{C}$ & 0.853000064 & 24.198001846 & 8.481000646 \\
$\mathrm{C}$ & 0.899000067 & 15.775001207 & 16.202001240 \\
$\mathrm{C}$ & 0.903000070 & 23.913001828 & 16.258000242 \\
$\mathrm{C}$ & 1.210000092 & 13.377001022 & 16.157001234 \\
$\mathrm{C}$ & 1.309000101 & 24.468001869 & 12.602000964 \\
$\mathrm{C}$ & 1.759000136 & 14.642001119 & 16.139001233 \\
$\mathrm{C}$ & 2.689000205 & 22.688001731 & 8.865000678 \\
$\mathrm{C}$ & 2.960000224 & 23.011001755 & 12.789000975 \\
$\mathrm{C}$ & 3.207000246 & 18.353002403 & 13.028000995 \\
$\mathrm{C}$ & 3.207000246 & 18.548001415 & 9.745000742 \\
$\mathrm{C}$ & 3.217000246 & 19.891002520 & 16.202001240 \\
$\mathrm{C}$ & 3.400000259 & 13.297001016 & 16.122001229 \\
$\mathrm{C}$ & 3.696000284 & 16.303000242 & 8.865000678 \\
$\mathrm{C}$ & 4.019000308 & 16.032001223 & 12.789000975 \\
$\mathrm{C}$ & 4.350000333 & 20.751000583 & 16.139001233 \\
$\mathrm{C}$ & 4.455000339 & 18.998000449 & 9.192000700 \\
$\mathrm{C}$ & 4.549000348 & 18.692000429 & 12.760000977 \\
$\mathrm{C}$ & 4.921000376 & 18.089002383 & 16.258000242 \\
$\mathrm{C}$ & 5.206000399 & 18.139001383 & 8.481000646 \\
$\mathrm{C}$ & 5.476000416 & 17.683002352 & 12.602000964 \\
$\mathrm{C}$ & 5.615000431 & 20.202001545 & 16.157001234 \\
$\mathrm{C}$ & 5.695000437 & 22.392001711 & 16.122001229 \\
$\mathrm{C}$ & 6.406000491 & 19.668000501 & 12.427000947 \\
$\mathrm{C}$ & -0.006000002 & 23.447002792 & 9.192000700 \\
$\mathrm{C}$ & -0.300000023 & 14.443001104 & 12.760000977 \\
$\mathrm{C}$ & -0.444000032 & 15.785001207 & 9.745000742 \\
$\mathrm{C}$ & -0.639000049 & 15.785001207 & 13.028000995 \\
$\mathrm{C}$ & -0.676000054 & 25.398002938 & 12.427000947 \\
$\mathrm{C}$ & -0.853000064 & 13.786001054 & 8.481000646 \\
$\mathrm{C}$ & -0.899000067 & 22.209001698 & 16.202001240 \\
$\mathrm{C}$ & -0.903000070 & 14.071001077 & 16.258000242 \\
$\mathrm{C}$ & -1.210000092 & 24.607001878 & 16.157001234 \\
$\mathrm{C}$ & -1.309000101 & 13.516001031 & 12.602000964 \\
$\mathrm{C}$ & -1.759000136 & 23.342000780 & 16.139001233 \\
& -2.689000205 & 15.296001169 & 8.865000678 \\
\hline & & &
\end{tabular}

Continued on next page 
Table S5 - Continued from previous page

\begin{tabular}{|c|c|c|c|}
\hline $\mathrm{C}$ & -2.960000224 & 14.973001145 & 12.789000975 \\
\hline $\mathrm{C}$ & -3.207000246 & 19.436002485 & 00742 \\
\hline $\mathrm{C}$ & -3.207000246 & 19.631002497 & 13.028000995 \\
\hline $\mathrm{C}$ & -3.217000246 & 18.093001380 & 16.20 \\
\hline $\mathrm{C}$ & -3.400000259 & 24.687001884 & 16.122001229 \\
\hline $\mathrm{C}$ & -3.696000284 & 21.681001657 & 8.865000678 \\
\hline $\mathrm{C}$ & -4.019000308 & 21.952001677 & 12.789000975 \\
\hline $\mathrm{C}$ & -4.350000333 & 17.2 & 16.1 \\
\hline $\mathrm{C}$ & -4.455000339 & 18.986001450 & 9.1920 \\
\hline $\mathrm{C}$ & -4.549000348 & 19.29200 & 12.760 \\
\hline $\mathrm{C}$ & -4.921000376 & 19.895001517 & 16.258000242 \\
\hline $\mathrm{C}$ & -5.206000399 & 19.845000517 & 8.481000646 \\
\hline $\mathrm{C}$ & -5.476000416 & 20.301002548 & 12.602 \\
\hline $\mathrm{C}$ & -5.61 & 17.7 & 16.15 \\
\hline $\mathrm{C}$ & -5.69 & 15. & 16.1 \\
\hline $\mathrm{C}$ & -6.40 & 18.31600 & 12.42 \\
\hline $\mathrm{H}$ & 0.501000037 & 25.122001918 & 8.046000617 \\
\hline $\mathrm{H}$ & 1.02 & 14.2 & 9.34 \\
\hline $\mathrm{H}$ & 1.09 & 26.5 & 12.2 \\
\hline $\mathrm{H}$ & 1.15 & 21. & 16.3 \\
\hline $\mathrm{H}$ & 1.43 & 904 & 12.28 \\
\hline $\mathrm{H}$ & 2.066000157 & 16.874002291 & 9.949000762 \\
\hline $\mathrm{H}$ & 0159 & 16.6 & 13.16 \\
\hline $\mathrm{H}$ & 2.11 & 21.0 & 9.94 \\
\hline $\mathrm{H}$ & 2.24 & 11.5 & 16.26 \\
\hline $\mathrm{H}$ & 2.36 & 21.0 & 1006 \\
\hline $\mathrm{H}$ & 2.592000200 & 25.027001912 & 16.285001241 \\
\hline $\mathrm{H}$ & 0204 & 14.511001106 & 12.93 \\
\hline $\mathrm{H}$ & 2.75 & 24.40185 & 4418 \\
\hline $\mathrm{H}$ & 2.823000214 & 23.276000778 & 16.323001248 \\
\hline $\mathrm{H}$ & 2.857000217 & 17.842000361 & 16.330001247 \\
\hline $\mathrm{H}$ & 4.284000325 & 16.169002233 & 16.323001248 \\
\hline $\mathrm{H}$ & 4.372 & 001070 & 12.627000964 \\
\hline $\mathrm{H}$ & 4.400000 & 12.892000987 & 16.070001230 \\
\hline $\mathrm{H}$ & 4.481000341 & 21.688001656 & 12.937000987 \\
\hline $\mathrm{H}$ & 4.786000364 & 20.015000528 & 9.346000715 \\
\hline $\mathrm{H}$ & 4.944 & 23.364001785 & 12.627000964 \\
\hline $\mathrm{H}$ & 5.409 & 16.232393805 & 7.716613847 \\
\hline $\mathrm{H}$ & 6.035000460 & 16.400001253 & 16.285001241 \\
\hline $\mathrm{H}$ & 6.100000466 & 23.392001786 & 16.070001230 \\
\hline $\mathrm{H}$ & 6.130000466 & 18.491000410 & 8.046000617 \\
\hline
\end{tabular}


Table S5 - Continued from previous page

\begin{tabular}{cccc}
\hline \hline $\mathrm{H}$ & 7.161000548 & 20.427001562 & 12.288000938 \\
$\mathrm{H}$ & 7.468222735 & 21.239800200 & 16.260676207 \\
$\mathrm{H}$ & 7.568674282 & 17.893501711 & 12.265385749 \\
$\mathrm{H}$ & -0.501000037 & 12.862000982 & 8.046000617 \\
$\mathrm{H}$ & -1.023000076 & 23.778001817 & 9.346000715 \\
$\mathrm{H}$ & -1.098499514 & 11.423327065 & 12.265385733 \\
$\mathrm{H}$ & -1.150000086 & 16.135001230 & 16.330001247 \\
$\mathrm{H}$ & -1.435000109 & 26.153001995 & 12.288000938 \\
$\mathrm{H}$ & -2.066000157 & 21.110002609 & 9.949000762 \\
$\mathrm{H}$ & -2.069000159 & 21.360002632 & 13.165001006 \\
$\mathrm{H}$ & -2.118000162 & 16.926002290 & 9.949000762 \\
$\mathrm{H}$ & -2.247797297 & 26.460223166 & 16.260676276 \\
$\mathrm{H}$ & -2.368000179 & 16.923001294 & 13.165001006 \\
$\mathrm{H}$ & -2.592000200 & 12.957000987 & 16.285001241 \\
$\mathrm{H}$ & -2.696000204 & 23.473001794 & 12.937000987 \\
$\mathrm{H}$ & -2.759609251 & 13.582143716 & 7.716614418 \\
$\mathrm{H}$ & -2.823000214 & 14.708001122 & 16.323001248 \\
$\mathrm{H}$ & -2.857000217 & 20.142001539 & 16.330001247 \\
$\mathrm{H}$ & -4.284000325 & 21.815002667 & 16.323001248 \\
$\mathrm{H}$ & -4.372000332 & 23.936002830 & 12.627000964 \\
$\mathrm{H}$ & -4.400000334 & 25.092000918 & 16.070001230 \\
$\mathrm{H}$ & -4.481000341 & 16.296001244 & 12.937000987 \\
$\mathrm{H}$ & -4.786000364 & 17.969001371 & 9.346000715 \\
$\mathrm{H}$ & -4.944000377 & 14.620001115 & 12.627000964 \\
$\mathrm{H}$ & -5.409857906 & 21.751612027 & 7.716614932 \\
$\mathrm{H}$ & -6.035000460 & 21.584001647 & 16.285001241 \\
$\mathrm{H}$ & -6.100000466 & 14.592001114 & 16.070001230 \\
$\mathrm{H}$ & -6.130000466 & 19.493001489 & 8.046000617 \\
$\mathrm{H}$ & -7.161000548 & 17.557000343 & 12.288000938 \\
$\mathrm{H}$ & -7.468222724 & 16.744204139 & 16.260676196 \\
$\mathrm{H}$ & -7.568674690 & 20.090499315 & 12.265385696 \\
$\mathrm{~N}$ & 0.104000009 & 24.963000909 & 16.199000239 \\
$\mathrm{~N}$ & 0.451000037 & 22.589002727 & 16.269000244 \\
$\mathrm{~N}$ & 0.669000050 & 25.668000961 & 12.402000947 \\
$\mathrm{~N}$ & 0.945000075 & 13.837001056 & 12.634000963 \\
$\mathrm{~N}$ & 1.771000135 & 21.928000673 & 9.545000730 \\
$\mathrm{~N}$ & 2.020000155 & 22.007001682 & 13.008000995 \\
$\mathrm{~N}$ & 2.170000167 & 23.830001822 & 8.287000631 \\
$\mathrm{~N}$ & 2.202000166 & 24.085000839 & 16.291001244 \\
& 2.281000175 & 12.521000956 & 16.194001239 \\
& 2.639000199 & 24.289001854 & 12.594000963 \\
\hline
\end{tabular}


Table S5 - Continued from previous page

\begin{tabular}{cccc}
\hline \hline $\mathrm{N}$ & 2.936000226 & 17.221002313 & 9.545000730 \\
$\mathrm{~N}$ & 3.015000230 & 16.972001297 & 13.008000995 \\
$\mathrm{~N}$ & 3.145000241 & 14.577001114 & 16.122001229 \\
$\mathrm{~N}$ & 3.597000275 & 18.541001416 & 16.269000244 \\
$\mathrm{~N}$ & 3.670000281 & 14.771001129 & 12.784000975 \\
$\mathrm{~N}$ & 4.221000324 & 22.662002729 & 12.784000975 \\
$\mathrm{~N}$ & 4.415000339 & 22.137000688 & 16.122001229 \\
$\mathrm{~N}$ & 4.838000369 & 16.822002286 & 8.287000631 \\
$\mathrm{~N}$ & 5.093000386 & 16.790002282 & 16.291001244 \\
$\mathrm{~N}$ & 5.155000396 & 19.937001522 & 12.634000963 \\
$\mathrm{~N}$ & 5.297000407 & 16.353002248 & 12.594000963 \\
$\mathrm{~N}$ & 5.971000456 & 18.888001443 & 16.199000239 \\
$\mathrm{~N}$ & 6.471000496 & 21.273002622 & 16.194001239 \\
$\mathrm{~N}$ & 6.676000508 & 18.323001398 & 12.402000947 \\
$\mathrm{~N}$ & -0.104000009 & 13.021000996 & 16.199000239 \\
$\mathrm{~N}$ & -0.451000037 & 15.395001178 & 16.269000244 \\
$\mathrm{~N}$ & -0.669000050 & 12.316000939 & 12.402000947 \\
$\mathrm{~N}$ & -0.945000075 & 24.147000843 & 12.634000963 \\
$\mathrm{~N}$ & -1.771000135 & 16.056001226 & 9.545000730 \\
$\mathrm{~N}$ & -2.020000155 & 15.977001223 & 13.008000995 \\
$\mathrm{~N}$ & -2.170000167 & 14.154001078 & 8.287000631 \\
$\mathrm{~N}$ & -2.202000166 & 13.899001061 & 16.291001244 \\
$\mathrm{~N}$ & -2.281000175 & 25.463000943 & 16.194001239 \\
$\mathrm{~N}$ & -2.639000199 & 13.695001046 & 12.594000963 \\
$\mathrm{~N}$ & -2.936000226 & 20.763001587 & 9.545000730 \\
$\mathrm{~N}$ & -3.015000230 & 21.012000602 & 13.008000995 \\
$\mathrm{~N}$ & -3.145000241 & 23.407001786 & 16.122001229 \\
$\mathrm{~N}$ & -3.597000275 & 19.443002484 & 16.269000244 \\
$\mathrm{~N}$ & -3.670000281 & 23.213000771 & 12.784000975 \\
$\mathrm{~N}$ & -4.221000324 & 15.322001171 & 12.784000975 \\
$\mathrm{~N}$ & -4.415000339 & 15.847001211 & 16.122001229 \\
$\mathrm{~N}$ & -4.838000369 & 21.162002614 & 8.287000631 \\
$\mathrm{~N}$ & -5.093000386 & 21.194001618 & 16.291001244 \\
$\mathrm{~N}$ & -5.155000396 & 18.047002378 & 12.634000963 \\
$\mathrm{~N}$ & -5.297000407 & 21.631002652 & 12.594000963 \\
$\mathrm{~N}$ & -5.971000456 & 19.096002456 & 16.199000239 \\
$\mathrm{~N}$ & -6.471000496 & 16.711001278 & 16.194001239 \\
$\mathrm{~N}$ & -6.676000508 & 19.661000502 & 12.402000947 \\
$\mathrm{O}$ & 0.111000008 & 16.723001277 & 13.274001015 \\
$\mathrm{O}$ & 0.282000022 & 16.669002274 & 10.225000781 \\
$\mathrm{O}$ & 1.209000090 & 16.942000292 & 16.233001242 \\
\hline & & Continued on next page
\end{tabular}


Table S5 - Continued from previous page

\begin{tabular}{cccc}
\hline \hline $\mathrm{O}$ & 2.050000155 & 20.201001542 & 16.233001242 \\
$\mathrm{O}$ & 2.269000176 & 19.103002461 & 13.274001015 \\
$\mathrm{O}$ & 2.323000179 & 19.274001474 & 10.225000781 \\
$\mathrm{O}$ & 3.387000258 & 15.117001154 & 8.792000671 \\
$\mathrm{O}$ & 3.875000293 & 22.379001710 & 8.792000671 \\
$\mathrm{O}$ & -0.111000008 & 21.261001623 & 13.274001015 \\
$\mathrm{O}$ & -0.282000022 & 21.315002627 & 10.225000781 \\
$\mathrm{O}$ & -1.209000090 & 21.042001608 & 16.233001242 \\
$\mathrm{O}$ & -2.050000155 & 17.783002358 & 16.233001242 \\
$\mathrm{O}$ & -2.269000176 & 18.881002439 & 13.274001015 \\
$\mathrm{O}$ & -2.323000179 & 18.710000431 & 10.225000781 \\
$\mathrm{O}$ & -3.387000258 & 22.867002746 & 8.792000671 \\
$\mathrm{O}$ & -3.875000293 & 15.605001190 & 8.792000671 \\
$\mathrm{Ba}$ & 0.016999999 & 18.992002453 & 10.424000796 \\
$\mathrm{Ba}$ & -0.021000002 & 19.059001457 & 14.812001131 \\
\hline
\end{tabular}


Table S6: The Cartesian coordinates of the structure 6 in $\AA$.

\begin{tabular}{lcrl}
\hline \hline $\mathrm{C}$ & -2.573340495 & -3.827553494 & -3.431845664 \\
$\mathrm{C}$ & -2.328340477 & -5.179553295 & -3.491845569 \\
$\mathrm{C}$ & -1.489340712 & -2.944553726 & -3.329845654 \\
$\mathrm{C}$ & -0.134341011 & -4.974553277 & -3.489845665 \\
$\mathrm{C}$ & -4.482340044 & -4.754553361 & -3.487845666 \\
$\mathrm{H}$ & 0.560309641 & -3.066547836 & -3.362661258 \\
$\mathrm{H}$ & -3.756207589 & -6.748775017 & -3.629551677 \\
$\mathrm{H}$ & -5.540339823 & -4.915553274 & -3.467845666 \\
$\mathrm{H}$ & 1.872658645 & -4.808553370 & -3.372845655 \\
$\mathrm{H}$ & 1.266386494 & -6.425229093 & -3.513097867 \\
$\mathrm{~N}$ & -0.274340922 & -3.616553575 & -3.391845658 \\
$\mathrm{~N}$ & -3.563340273 & -5.770553142 & -3.552845572 \\
$\mathrm{~N}$ & -1.149340790 & -5.817553146 & -3.521845569 \\
$\mathrm{~N}$ & -3.931340202 & -3.569553571 & -3.452845666 \\
$\mathrm{~N}$ & 1.101658782 & -5.440553214 & -3.455845662 \\
$\mathrm{O}$ & -1.522340714 & -1.712553931 & -3.294845654 \\
$\mathrm{C}$ & 1.509658716 & -4.413553436 & 3.483152965 \\
$\mathrm{C}$ & 2.734658411 & -5.017553284 & 3.578152971 \\
$\mathrm{C}$ & 1.441658709 & -3.028553729 & 3.297152951 \\
$\mathrm{C}$ & 3.894658196 & -3.143553640 & 3.402152962 \\
$\mathrm{C}$ & 1.109658783 & -6.491552995 & 3.705152881 \\
$\mathrm{H}$ & 2.755109212 & -1.447991712 & 3.200606943 \\
$\mathrm{H}$ & 3.135398040 & -7.074130440 & 3.974744802 \\
$\mathrm{H}$ & 0.606658847 & -7.433552766 & 3.772152890 \\
$\mathrm{H}$ & 4.978657978 & -1.444554013 & 3.250153047 \\
$\mathrm{H}$ & 5.900427950 & -2.897954319 & 3.446708163 \\
$\mathrm{~N}$ & 2.703658404 & -2.442553787 & 3.291152949 \\
$\mathrm{~N}$ & 2.468658487 & -6.351552983 & 3.792152891 \\
$\mathrm{~N}$ & 3.961658201 & -4.448553442 & 3.568152971 \\
$\mathrm{~N}$ & 0.491658937 & -5.354553211 & 3.531152972 \\
$\mathrm{~N}$ & 5.016657985 & -2.437553787 & 3.363152959 \\
$\mathrm{O}$ & 0.439658932 & -2.331553880 & 3.156153043 \\
$\mathrm{C}$ & 2.573658498 & 3.827444891 & -3.431845664 \\
$\mathrm{C}$ & 2.328658476 & 5.179444596 & -3.491845569 \\
$\mathrm{C}$ & 1.489658716 & 2.944445123 & -3.329845654 \\
$\mathrm{C}$ & 0.134659010 & 4.974444680 & -3.489845665 \\
$\mathrm{C}$ & 4.482658042 & 4.754444763 & -3.487845666 \\
$\mathrm{H}$ & -0.559991642 & 3.066439232 & -3.362661258 \\
$\mathrm{H}$ & 3.756525588 & 6.748666313 & -3.629551677 \\
$\mathrm{H}$ & 5.540657822 & 4.915444676 & -3.467845666 \\
\hline & & Continued \\
& & & \\
& & & \\
& &
\end{tabular}


Table S6 - Continued from previous page

\begin{tabular}{cccc}
\hline \hline $\mathrm{H}$ & -1.870340643 & 4.810444665 & -3.350845656 \\
$\mathrm{H}$ & -1.267465095 & 6.423354790 & -3.535153769 \\
$\mathrm{~N}$ & 0.274658920 & 3.616444977 & -3.391845658 \\
$\mathrm{~N}$ & 3.563658271 & 5.770444538 & -3.552845572 \\
$\mathrm{~N}$ & 1.149658788 & 5.817444543 & -3.521845569 \\
$\mathrm{~N}$ & 3.931658201 & 3.569444973 & -3.452845666 \\
$\mathrm{~N}$ & -1.101340783 & 5.440444616 & -3.455845662 \\
$\mathrm{O}$ & 1.522658717 & 1.712445333 & -3.294845654 \\
$\mathrm{C}$ & -1.509340718 & 4.413444838 & 3.483152965 \\
$\mathrm{C}$ & -2.734340408 & 5.017444681 & 3.578152971 \\
$\mathrm{C}$ & -1.441340711 & 3.028445031 & 3.297152951 \\
$\mathrm{C}$ & -3.894340198 & 3.143445042 & 3.402152962 \\
$\mathrm{C}$ & -1.109340784 & 6.491444397 & 3.705152881 \\
$\mathrm{H}$ & -2.754791209 & 1.447883109 & 3.200606943 \\
$\mathrm{H}$ & -3.135080041 & 7.074021843 & 3.974744802 \\
$\mathrm{H}$ & -0.606340849 & 7.433444169 & 3.772152890 \\
$\mathrm{H}$ & -4.978339980 & 1.443445408 & 3.263153048 \\
$\mathrm{H}$ & -5.900430251 & 2.899393522 & 3.433776960 \\
$\mathrm{~N}$ & -2.703340405 & 2.442445184 & 3.291152949 \\
$\mathrm{~N}$ & -2.468340489 & 6.351444385 & 3.792152891 \\
$\mathrm{~N}$ & -3.961340202 & 4.448444738 & 3.568152971 \\
$\mathrm{~N}$ & -0.491340938 & 5.354444608 & 3.531152972 \\
$\mathrm{~N}$ & -5.016339981 & 2.437445184 & 3.363152959 \\
$\mathrm{O}$ & -0.439340934 & 2.331445176 & 3.156153043 \\
$\mathrm{C}$ & 3.827658192 & -2.573553795 & -3.431845664 \\
$\mathrm{C}$ & 5.179657897 & -2.328553878 & -3.491845569 \\
$\mathrm{C}$ & 2.944658423 & -1.489554013 & -3.329845654 \\
$\mathrm{C}$ & 4.974657980 & -0.134554312 & -3.489845665 \\
$\mathrm{C}$ & 4.754658064 & -4.482553445 & -3.487845666 \\
$\mathrm{H}$ & 3.066652533 & 0.560096345 & -3.362661258 \\
$\mathrm{H}$ & 6.748879714 & -3.756420885 & -3.629551677 \\
$\mathrm{H}$ & 4.915657977 & -5.540553225 & -3.467845666 \\
$\mathrm{H}$ & 4.810657966 & 1.870445340 & -3.342845655 \\
$\mathrm{H}$ & 6.422792989 & 1.268201895 & -3.543038271 \\
$\mathrm{~N}$ & 3.616658278 & -0.274554223 & -3.391845658 \\
$\mathrm{~N}$ & 5.770657839 & -3.563553574 & -3.552845572 \\
$\mathrm{~N}$ & 5.817657844 & -1.149554085 & -3.521845569 \\
$\mathrm{~N}$ & 3.569658274 & -3.931553498 & -3.452845666 \\
$\mathrm{~N}$ & 5.440657917 & 1.101445486 & -3.455845662 \\
$\mathrm{O}$ & 1.712658629 & -1.522554014 & -3.294845654 \\
& 4.413658139 & 1.509445415 & 3.483152965 \\
\hline & & Continued & on next page
\end{tabular}


Table S6 - Continued from previous page

\begin{tabular}{cccc}
\hline $\mathrm{C}$ & 5.017657982 & 2.734445111 & 3.578152971 \\
$\mathrm{C}$ & 3.028658332 & 1.441445409 & 3.297152951 \\
$\mathrm{C}$ & 3.143658338 & 3.894444895 & 3.402152962 \\
$\mathrm{C}$ & 6.491657698 & 1.109445487 & 3.705152881 \\
$\mathrm{H}$ & 1.448096410 & 2.754895912 & 3.200606943 \\
$\mathrm{H}$ & 7.074235138 & 3.135184739 & 3.974744802 \\
$\mathrm{H}$ & 7.433657469 & 0.606445547 & 3.772152890 \\
$\mathrm{H}$ & 1.446658710 & 4.979444680 & 3.232153046 \\
$\mathrm{H}$ & 2.895989420 & 5.899405849 & 3.464700665 \\
$\mathrm{~N}$ & 2.442658485 & 2.703445108 & 3.291152949 \\
$\mathrm{~N}$ & 6.351657686 & 2.468445186 & 3.792152891 \\
$\mathrm{~N}$ & 4.448658139 & 3.961444900 & 3.568152971 \\
$\mathrm{~N}$ & 5.354657909 & 0.491445636 & 3.531152972 \\
$\mathrm{~N}$ & 2.437658485 & 5.016444684 & 3.363152959 \\
$\mathrm{O}$ & 2.331658477 & 0.439445631 & 3.156153043 \\
$\mathrm{C}$ & -3.827340193 & 2.573445198 & -3.431845664 \\
$\mathrm{C}$ & -5.179339893 & 2.328445180 & -3.491845569 \\
$\mathrm{C}$ & -2.944340425 & 1.489445415 & -3.329845654 \\
$\mathrm{C}$ & -4.974339982 & 0.134445709 & -3.489845665 \\
$\mathrm{C}$ & -4.754340065 & 4.482444741 & -3.487845666 \\
$\mathrm{H}$ & -3.066334535 & -0.560204943 & -3.362661258 \\
$\mathrm{H}$ & -6.748561716 & 3.756312186 & -3.629551677 \\
$\mathrm{H}$ & -4.915339973 & 5.540444521 & -3.467845666 \\
$\mathrm{H}$ & -4.808339968 & -1.872553942 & -3.375845656 \\
$\mathrm{H}$ & -6.425214588 & -1.266100897 & -3.510120266 \\
$\mathrm{~N}$ & -3.616340274 & 0.274445620 & -3.391845658 \\
$\mathrm{~N}$ & -5.770339841 & 3.563444971 & -3.552845572 \\
$\mathrm{~N}$ & -5.817339845 & 1.149445487 & -3.521845569 \\
$\mathrm{~N}$ & -3.569340275 & 3.931444900 & -3.452845666 \\
$\mathrm{~N}$ & -5.440339913 & -1.101554084 & -3.455845662 \\
$\mathrm{O}$ & -1.712340630 & 1.522445417 & -3.294845654 \\
$\mathrm{C}$ & -4.413340135 & -1.509554013 & 3.483152965 \\
$\mathrm{C}$ & -5.017339983 & -2.734553708 & 3.578152971 \\
$\mathrm{C}$ & -3.028340333 & -1.441554012 & 3.297152951 \\
$\mathrm{C}$ & -3.143340339 & -3.894553498 & 3.402152962 \\
$\mathrm{C}$ & -6.491339694 & -1.109554085 & 3.705152881 \\
$\mathrm{H}$ & -1.447778412 & -2.755004509 & 3.200606943 \\
$\mathrm{H}$ & -7.073917140 & -3.135293342 & 3.974744802 \\
$\mathrm{H}$ & -7.433339466 & -0.606554144 & 3.772152890 \\
$\mathrm{H}$ & -1.442340708 & -4.978553281 & 3.275153053 \\
& -2.900536323 & -5.900866553 & 3.421799160 \\
\hline & & Continued & \\
& & & \\
\hline & &
\end{tabular}


Table S6 - Continued from previous page

\begin{tabular}{cccc}
\hline \hline $\mathrm{N}$ & -2.442340486 & -2.703553807 & 3.291152949 \\
$\mathrm{~N}$ & -6.351339688 & -2.468553789 & 3.792152891 \\
$\mathrm{~N}$ & -4.448340141 & -3.961553503 & 3.568152971 \\
$\mathrm{~N}$ & -5.354339910 & -0.491554239 & 3.531152972 \\
$\mathrm{~N}$ & -2.437340486 & -5.016553282 & 3.363152959 \\
$\mathrm{O}$ & -2.331340478 & -0.439554234 & 3.156153043 \\
$\mathrm{C}$ & -1.506340717 & -4.504553343 & -0.073846304 \\
$\mathrm{C}$ & -3.717340282 & -3.799553493 & 0.087153609 \\
$\mathrm{C}$ & -4.184340120 & -5.141553293 & 0.138153611 \\
$\mathrm{C}$ & -3.281340352 & -6.105553064 & 0.052153704 \\
$\mathrm{H}$ & -1.303527202 & -6.565211599 & -0.253090121 \\
$\mathrm{H}$ & -3.596340274 & -7.126552845 & 0.110153610 \\
$\mathrm{H}$ & -5.226339898 & -5.363553209 & 0.242153621 \\
$\mathrm{H}$ & -4.268340124 & -1.849553941 & 0.023153701 \\
$\mathrm{H}$ & -5.569339827 & -2.982553727 & 0.184153614 \\
$\mathrm{~N}$ & -1.956340651 & -5.821553144 & -0.108846310 \\
$\mathrm{~N}$ & -2.416340484 & -3.506553569 & -0.027846302 \\
$\mathrm{~N}$ & -4.591340053 & -2.793553712 & 0.099153608 \\
$\mathrm{O}$ & -0.293340920 & -4.295553429 & -0.167846313 \\
$\mathrm{C}$ & 1.506658715 & 4.504444746 & -0.073846304 \\
$\mathrm{C}$ & 3.717658286 & 3.799444890 & 0.087153609 \\
$\mathrm{C}$ & 4.184658119 & 5.141444595 & 0.138153611 \\
$\mathrm{C}$ & 3.281658350 & 6.105444466 & 0.052153704 \\
$\mathrm{H}$ & 1.303845200 & 6.565103002 & -0.253090121 \\
$\mathrm{H}$ & 3.596658273 & 7.126444242 & 0.110153610 \\
$\mathrm{H}$ & 5.226657902 & 5.363444611 & 0.242153621 \\
$\mathrm{H}$ & 4.268658128 & 1.849445343 & 0.023153701 \\
$\mathrm{H}$ & 5.569657825 & 2.982445129 & 0.184153614 \\
$\mathrm{~N}$ & 1.956658649 & 5.821444546 & -0.108846310 \\
$\mathrm{~N}$ & 2.416658483 & 3.506444966 & -0.027846302 \\
$\mathrm{~N}$ & 4.591658051 & 2.793445114 & 0.099153608 \\
$\mathrm{O}$ & 0.293658924 & 4.295444826 & -0.167846313 \\
$\mathrm{C}$ & 4.504658046 & -1.506554017 & -0.073846304 \\
$\mathrm{C}$ & 3.799658190 & -3.717553583 & 0.087153609 \\
$\mathrm{C}$ & 5.141657991 & -4.184553421 & 0.138153611 \\
$\mathrm{C}$ & 6.105657767 & -3.281553652 & 0.052153704 \\
$\mathrm{H}$ & 6.565316302 & -1.303740497 & -0.253090121 \\
$\mathrm{H}$ & 7.126657543 & -3.596553575 & 0.110153610 \\
$\mathrm{H}$ & 5.363657912 & -5.226553199 & 0.242153621 \\
$\mathrm{H}$ & 1.849658639 & -4.268553425 & 0.023153701 \\
$\mathrm{H}$ & 2.982658430 & -5.569553128 & 0.184153614 \\
\hline & & &
\end{tabular}


Table S6 - Continued from previous page

\begin{tabular}{cccc}
\hline $\mathrm{N}$ & 5.821657847 & -1.956553951 & -0.108846310 \\
$\mathrm{~N}$ & 3.506658267 & -2.416553785 & -0.027846302 \\
$\mathrm{~N}$ & 2.793658415 & -4.591553348 & 0.099153608 \\
$\mathrm{O}$ & 4.295658127 & -0.293554221 & -0.167846313 \\
$\mathrm{C}$ & -4.504340043 & 1.506445414 & -0.073846304 \\
$\mathrm{C}$ & -3.799340192 & 3.717444885 & 0.087153609 \\
$\mathrm{C}$ & -5.141339993 & 4.184444818 & 0.138153611 \\
$\mathrm{C}$ & -6.105339769 & 3.281445049 & 0.052153704 \\
$\mathrm{H}$ & -6.564998299 & 1.303631899 & -0.253090121 \\
$\mathrm{H}$ & -7.126339544 & 3.596444977 & 0.110153610 \\
$\mathrm{H}$ & -5.363339908 & 5.226444601 & 0.242153621 \\
$\mathrm{H}$ & -1.849340640 & 4.268444827 & 0.023153701 \\
$\mathrm{H}$ & -2.982340426 & 5.569444525 & 0.184153614 \\
$\mathrm{~N}$ & -5.821339843 & 1.956445248 & -0.108846310 \\
$\mathrm{~N}$ & -3.506340268 & 2.416445187 & -0.027846302 \\
$\mathrm{~N}$ & -2.793340411 & 4.591444750 & 0.099153608 \\
$\mathrm{O}$ & -4.295340128 & 0.293445623 & -0.167846313 \\
$\mathrm{H}$ & 0.307529824 & -2.773405214 & -0.095693408 \\
$\mathrm{H}$ & 1.764432733 & -2.021227555 & -0.075694207 \\
$\mathrm{O}$ & 0.760658857 & -1.869553941 & -0.068846304 \\
$\mathrm{H}$ & -0.307123426 & 2.773223214 & -0.095319010 \\
$\mathrm{H}$ & -1.764141537 & 2.021236752 & -0.075325804 \\
$\mathrm{O}$ & -0.760340858 & 1.869445344 & -0.068846304 \\
$\mathrm{H}$ & 2.773429911 & 0.307220922 & -0.095409907 \\
$\mathrm{H}$ & 2.021382456 & 1.764203033 & -0.075367308 \\
$\mathrm{O}$ & 1.869658644 & 0.760445556 & -0.068846304 \\
$\mathrm{H}$ & -2.773220410 & -0.307451024 & -0.095557806 \\
$\mathrm{H}$ & -2.021039856 & -1.764368633 & -0.075651608 \\
$\mathrm{O}$ & -1.869340641 & -0.760554159 & -0.068846304 \\
$\mathrm{Ba}$ & -0.000341002 & -0.000554303 & 1.904153243 \\
$\mathrm{Ba}$ & -0.000341002 & 0.000445700 & -1.950845949 \\
\hline & & & \\
\hline
\end{tabular}


Table S7: The Cartesian coordinates of the optimized guanine octet chelated with $\mathrm{Na}^{+}$at $\mathrm{B}$ LYP-D3/def2-SVP level in Å.

\begin{tabular}{cccc}
\hline \hline $\mathrm{O}$ & -0.397925183 & -2.279477875 & 1.336947319 \\
$\mathrm{~N}$ & -2.705503830 & -2.345399158 & 1.520433890 \\
$\mathrm{C}$ & -3.919577951 & -3.015467326 & 1.616216223 \\
$\mathrm{~N}$ & -4.017645027 & -4.355454458 & 1.678617471 \\
$\mathrm{C}$ & -2.826993166 & -4.975580195 & 1.642061637 \\
$\mathrm{C}$ & -1.539370950 & -4.396138728 & 1.564123667 \\
$\mathrm{C}$ & -1.437317645 & -2.965353475 & 1.469534448 \\
$\mathrm{~N}$ & -2.593358337 & -6.345035841 & 1.652541981 \\
$\mathrm{C}$ & -1.216259711 & -6.531257714 & 1.582222515 \\
$\mathrm{~N}$ & -0.561393867 & -5.382213885 & 1.530103589 \\
$\mathrm{H}$ & -5.092103860 & -1.228346219 & 1.577635297 \\
$\mathrm{H}$ & -5.918545216 & -2.801626387 & 1.701193196 \\
$\mathrm{H}$ & -2.680760933 & -1.295987986 & 1.460417275 \\
$\mathrm{H}$ & -3.311044978 & -7.067487539 & 1.723704311 \\
$\mathrm{H}$ & -0.763012440 & -7.528696535 & 1.568939665 \\
$\mathrm{~N}$ & -2.272404190 & 5.052299848 & 1.628950464 \\
$\mathrm{O}$ & -2.277748466 & 0.400180733 & 1.335997137 \\
$\mathrm{~N}$ & -2.343506472 & 2.707927372 & 1.518491285 \\
$\mathrm{C}$ & -3.013644632 & 3.921965488 & 1.613065711 \\
$\mathrm{~N}$ & -4.353557725 & 4.020255471 & 1.674675190 \\
$\mathrm{C}$ & -4.973891899 & 2.829520745 & 1.638588536 \\
$\mathrm{C}$ & -4.394299586 & 1.541908725 & 1.561862965 \\
$\mathrm{C}$ & -2.963570472 & 1.439715188 & 1.467879319 \\
$\mathrm{~N}$ & -6.343062200 & 2.595750211 & 1.648419000 \\
$\mathrm{C}$ & -6.529360870 & 1.218918777 & 1.578975225 \\
$\mathrm{~N}$ & -5.380389205 & 0.563858771 & 1.528025442 \\
$\mathrm{H}$ & -1.226432697 & 5.094735236 & 1.574803804 \\
$\mathrm{H}$ & -2.799782235 & 5.921059520 & 1.696578360 \\
$\mathrm{H}$ & -1.294053146 & 2.683149362 & 1.459071237 \\
$\mathrm{H}$ & -7.065781344 & 3.313727773 & 1.719126768 \\
$\mathrm{H}$ & -7.526793301 & 0.765367110 & 1.565006094 \\
$\mathrm{~N}$ & 5.053781135 & 2.275424133 & 1.632037516 \\
$\mathrm{O}$ & 0.401979799 & 2.280525811 & 1.336121652 \\
$\mathrm{~N}$ & 2.709535342 & 2.346354672 & 1.519966942 \\
$\mathrm{C}$ & 3.923651145 & 3.016545420 & 1.615216209 \\
$\mathrm{~N}$ & 4.021898438 & 4.356481589 & 1.676417406 \\
$\mathrm{C}$ & 2.831237439 & 4.976860412 & 1.639092767 \\
$\mathrm{C}$ & 1.543669291 & 4.397295368 & 1.561479899 \\
$\mathrm{C}$ & 1.441377935 & 2.966555912 & 1.468167672 \\
\hline & & &
\end{tabular}

Continued on next page 
Table S7 - Continued from previous page

\begin{tabular}{ccrc}
\hline \hline $\mathrm{N}$ & 2.597737627 & 6.346098994 & 1.648436250 \\
$\mathrm{C}$ & 1.220786926 & 6.532501219 & 1.577994979 \\
$\mathrm{~N}$ & 0.565717153 & 5.383471402 & 1.526735452 \\
$\mathrm{H}$ & 5.096147979 & 1.229256388 & 1.580202048 \\
$\mathrm{H}$ & 5.922649570 & 2.802633505 & 1.701397869 \\
$\mathrm{H}$ & 2.684725049 & 1.296975433 & 1.461143541 \\
$\mathrm{H}$ & 3.315447908 & 7.068839550 & 1.719191497 \\
$\mathrm{H}$ & 0.767565317 & 7.529808399 & 1.564228778 \\
$\mathrm{~N}$ & 2.276566981 & -5.051290537 & 1.634376506 \\
$\mathrm{O}$ & 2.281956759 & -0.399366050 & 1.338341856 \\
$\mathrm{~N}$ & 2.347629093 & -2.706901013 & 1.522214678 \\
$\mathrm{C}$ & 3.017642905 & -3.920875104 & 1.619022563 \\
$\mathrm{~N}$ & 4.357412370 & -4.019029556 & 1.683294026 \\
$\mathrm{C}$ & 4.977760499 & -2.828278020 & 1.647768810 \\
$\mathrm{C}$ & 4.398259742 & -1.540785351 & 1.568851432 \\
$\mathrm{C}$ & 2.967707549 & -1.438658887 & 1.472058402 \\
$\mathrm{~N}$ & 6.347002523 & -2.594599488 & 1.660397654 \\
$\mathrm{C}$ & 6.533248781 & -1.217642180 & 1.590325247 \\
$\mathrm{~N}$ & 5.384504588 & -0.562782872 & 1.536292223 \\
$\mathrm{H}$ & 1.230527862 & -5.093785557 & 1.579035193 \\
$\mathrm{H}$ & 2.803803202 & -5.919820033 & 1.704847510 \\
$\mathrm{H}$ & 1.298310608 & -2.682188599 & 1.461313852 \\
$\mathrm{H}$ & 7.069581005 & -3.312298478 & 1.733363139 \\
$\mathrm{H}$ & 7.530791726 & -0.764191184 & 1.578955895 \\
$\mathrm{~N}$ & -5.049794901 & -2.274376303 & 1.632584549 \\
$\mathrm{~N}$ & -1.951282273 & -5.203353731 & -1.619780746 \\
$\mathrm{O}$ & 1.300377344 & -1.874273781 & -1.391508495 \\
$\mathrm{~N}$ & -0.261476745 & -3.576553797 & -1.543091755 \\
$\mathrm{C}$ & -0.631507530 & -4.915671560 & -1.603849163 \\
$\mathrm{~N}$ & 0.258705648 & -5.922818434 & -1.636598223 \\
$\mathrm{C}$ & 1.533580827 & -5.503924944 & -1.601882884 \\
$\mathrm{C}$ & 2.019505592 & -4.175864909 & -1.551889327 \\
$\mathrm{C}$ & 1.066383614 & -3.103054387 & -1.493474030 \\
$\mathrm{~N}$ & 2.675255054 & -6.294423753 & -1.585569434 \\
$\mathrm{C}$ & 3.770616546 & -5.439968973 & -1.524568599 \\
$\mathrm{~N}$ & 3.408317005 & -4.167090655 & -1.505812362 \\
$\mathrm{H}$ & -2.722551459 & -4.494555400 & -1.566304113 \\
$\mathrm{H}$ & -2.184681338 & -6.194339766 & -1.643687955 \\
$\mathrm{H}$ & -0.992789258 & -2.823499390 & -1.498818307 \\
$\mathrm{H}$ & 2.688972390 & -7.314511637 & -1.628311723 \\
$\mathrm{H}$ & 4.800356479 & -5.812616523 & -1.489419898 \\
\hline & & Continued & on $n+2 x t p a g e$
\end{tabular}


Table S7 - Continued from previous page

\begin{tabular}{cccc}
\hline \hline $\mathrm{N}$ & -5.205316400 & 1.948345104 & -1.621548665 \\
$\mathrm{O}$ & -1.876493750 & -1.303497330 & -1.394553514 \\
$\mathrm{~N}$ & -3.578675370 & 0.258407430 & -1.544716330 \\
$\mathrm{C}$ & -4.917828989 & 0.628605697 & -1.605321096 \\
$\mathrm{~N}$ & -5.924922559 & -0.261651889 & -1.637683948 \\
$\mathrm{C}$ & -5.506140704 & -1.536634426 & -1.603559601 \\
$\mathrm{C}$ & -4.178112843 & -2.022516801 & -1.554637456 \\
$\mathrm{C}$ & -3.105296252 & -1.069477599 & -1.496030593 \\
$\mathrm{~N}$ & -6.296791016 & -2.678224664 & -1.587104304 \\
$\mathrm{C}$ & -5.442148806 & -3.773696236 & -1.527315499 \\
$\mathrm{~N}$ & -4.169404049 & -3.411450425 & -1.509249216 \\
$\mathrm{H}$ & -4.496456841 & 2.719647599 & -1.568901611 \\
$\mathrm{H}$ & -6.196445970 & 2.181645060 & -1.645401683 \\
$\mathrm{H}$ & -2.825409419 & 0.989562279 & -1.500252896 \\
$\mathrm{H}$ & -7.316754939 & -2.691855433 & -1.629725530 \\
$\mathrm{H}$ & -5.814945108 & -4.803357427 & -1.492591830 \\
$\mathrm{~N}$ & 1.946770612 & 5.202461488 & -1.621826913 \\
$\mathrm{O}$ & -1.304823323 & 1.873285013 & -1.395709971 \\
$\mathrm{~N}$ & 0.257004858 & 3.575670859 & -1.545636552 \\
$\mathrm{C}$ & 0.626869283 & 4.914873218 & -1.606575685 \\
$\mathrm{~N}$ & -0.263342343 & 5.921808304 & -1.640220988 \\
$\mathrm{C}$ & -1.538387395 & 5.502792509 & -1.607040250 \\
$\mathrm{C}$ & -2.024013572 & 4.174766337 & -1.557793651 \\
$\mathrm{C}$ & -1.070924038 & 3.102018709 & -1.497789834 \\
$\mathrm{~N}$ & -2.679986272 & 6.293203793 & -1.592081565 \\
$\mathrm{C}$ & -3.775419979 & 5.438600406 & -1.532714589 \\
$\mathrm{~N}$ & -3.412993080 & 4.165868284 & -1.513524750 \\
$\mathrm{H}$ & 2.717806470 & 4.493614730 & -1.568535951 \\
$\mathrm{H}$ & 2.180139605 & 6.193565496 & -1.645498871 \\
$\mathrm{H}$ & 0.988199391 & 2.822704990 & -1.500112258 \\
$\mathrm{H}$ & -2.693818070 & 7.313298809 & -1.634738625 \\
$\mathrm{H}$ & -4.805192876 & 5.811245118 & -1.499335328 \\
$\mathrm{~N}$ & 5.201532625 & -1.949918002 & -1.613157703 \\
$\mathrm{O}$ & 1.871845874 & 1.301467495 & -1.393944651 \\
$\mathrm{~N}$ & 3.574530034 & -0.260248077 & -1.541250501 \\
$\mathrm{C}$ & 4.913886155 & -0.630107831 & -1.599893983 \\
$\mathrm{~N}$ & 5.920663065 & 0.260259960 & -1.633152129 \\
$\mathrm{C}$ & 5.501405709 & 1.535252448 & -1.602016888 \\
$\mathrm{C}$ & 4.173313406 & 2.020807712 & -1.554695055 \\
$\mathrm{C}$ & 3.100744942 & 1.067570058 & -1.494862596 \\
$\mathrm{~N}$ & 6.291803380 & 2.677094734 & -1.587546084 \\
\hline & & Continued & on $n$ ext page
\end{tabular}


Table S7 - Continued from previous page

\begin{tabular}{cccc}
\hline \hline $\mathrm{C}$ & 5.436763110 & 3.772404464 & -1.530024687 \\
$\mathrm{~N}$ & 4.164135180 & 3.409787349 & -1.511745191 \\
$\mathrm{H}$ & 4.492697160 & -2.720979862 & -1.560457427 \\
$\mathrm{H}$ & 6.192841599 & -2.183233434 & -1.635307862 \\
$\mathrm{H}$ & 2.821523759 & -0.991357606 & -1.495922388 \\
$\mathrm{H}$ & 7.311783058 & 2.690980943 & -1.629122126 \\
$\mathrm{H}$ & 5.809258148 & 4.802307745 & -1.496843166 \\
$\mathrm{Na}$ & 0.000627840 & -0.000071464 & -0.218250027 \\
\hline
\end{tabular}


Table S8: The Cartesian coordinates of the optimized guanine octet chelated with $\mathrm{Ca}^{2+}$ at BLYP-D3/def2-SVP level in Å.

\begin{tabular}{llrl}
\hline \hline $\mathrm{O}$ & -0.454708038 & -2.143719751 & 1.285730415 \\
$\mathrm{~N}$ & -2.752371806 & -2.289658869 & 1.532616995 \\
$\mathrm{C}$ & -3.952122926 & -2.995009580 & 1.638657576 \\
$\mathrm{~N}$ & -4.002722014 & -4.334297017 & 1.713575199 \\
$\mathrm{C}$ & -2.798301323 & -4.917813896 & 1.668567456 \\
$\mathrm{C}$ & -1.525969841 & -4.299044331 & 1.556649094 \\
$\mathrm{C}$ & -1.478986940 & -2.872860534 & 1.454079996 \\
$\mathrm{~N}$ & -2.528267424 & -6.277541107 & 1.691965514 \\
$\mathrm{C}$ & -1.152474110 & -6.428322820 & 1.592127662 \\
$\mathrm{~N}$ & -0.526244791 & -5.264689308 & 1.509631368 \\
$\mathrm{H}$ & -5.131893187 & -1.236334385 & 1.587268428 \\
$\mathrm{H}$ & -5.953857273 & -2.823532453 & 1.762754418 \\
$\mathrm{H}$ & -2.743755848 & -1.244559460 & 1.470025402 \\
$\mathrm{H}$ & -3.224860137 & -7.018983120 & 1.789796689 \\
$\mathrm{H}$ & -0.672659281 & -7.413551721 & 1.582995928 \\
$\mathrm{~N}$ & -2.285497333 & 5.095877140 & 1.645190618 \\
$\mathrm{O}$ & -2.144115168 & 0.453888578 & 1.285251737 \\
$\mathrm{~N}$ & -2.290037594 & 2.751504898 & 1.533297170 \\
$\mathrm{C}$ & -2.995403500 & 3.951244803 & 1.640043517 \\
$\mathrm{~N}$ & -4.334676772 & 4.001720532 & 1.715204037 \\
$\mathrm{C}$ & -4.918180788 & 2.797404789 & 1.669663990 \\
$\mathrm{C}$ & -4.299372818 & 1.525096171 & 1.557208527 \\
$\mathrm{C}$ & -2.873214858 & 1.478121123 & 1.454313951 \\
$\mathrm{~N}$ & -6.277967036 & 2.527362433 & 1.692612302 \\
$\mathrm{C}$ & -6.428717524 & 1.151683332 & 1.591943551 \\
$\mathrm{~N}$ & -5.265085521 & 0.525411524 & 1.509420565 \\
$\mathrm{H}$ & -1.236995540 & 5.131248182 & 1.587653043 \\
$\mathrm{H}$ & -2.823972193 & 5.953026971 & 1.764665960 \\
$\mathrm{H}$ & -1.244892247 & 2.743152661 & 1.470235822 \\
$\mathrm{H}$ & -7.019428164 & 3.223864684 & 1.790462448 \\
$\mathrm{H}$ & -7.413878790 & 0.671781054 & 1.582160013 \\
$\mathrm{~N}$ & 5.096031720 & 2.286918014 & 1.638848715 \\
$\mathrm{O}$ & 0.453746952 & 2.144844692 & 1.284059011 \\
$\mathrm{~N}$ & 2.751637561 & 2.291044817 & 1.529781365 \\
$\mathrm{C}$ & 3.951271522 & 2.996784731 & 1.634481535 \\
$\mathrm{~N}$ & 4.001627343 & 4.336112082 & 1.708282504 \\
$\mathrm{C}$ & 2.797170318 & 4.919486167 & 1.663907816 \\
$\mathrm{C}$ & 1.524925454 & 4.300445544 & 1.553322419 \\
$\mathrm{C}$ & 1.478118611 & 2.874123362 & 1.451545570 \\
\hline & & Continued on next page
\end{tabular}


Table S8 - Continued from previous page

\begin{tabular}{ccrc}
\hline \hline $\mathrm{N}$ & 2.526884716 & 6.279200801 & 1.687006095 \\
$\mathrm{C}$ & 1.151024001 & 6.429741931 & 1.588319323 \\
$\mathrm{~N}$ & 0.524861878 & 5.265927665 & 1.506729364 \\
$\mathrm{H}$ & 5.130911057 & 1.238269273 & 1.585654293 \\
$\mathrm{H}$ & 5.953272222 & 2.825586762 & 1.757079266 \\
$\mathrm{H}$ & 2.743227415 & 1.245713651 & 1.468121543 \\
$\mathrm{H}$ & 3.223419336 & 7.020744438 & 1.784057570 \\
$\mathrm{H}$ & 0.670900016 & 7.414821569 & 1.579462747 \\
$\mathrm{~N}$ & 2.284035771 & -5.094331743 & 1.649065559 \\
$\mathrm{O}$ & 2.143618518 & -0.452808368 & 1.285545821 \\
$\mathrm{~N}$ & 2.288909733 & -2.750154428 & 1.535617136 \\
$\mathrm{C}$ & 2.994032836 & -3.949776652 & 1.644404510 \\
$\mathrm{~N}$ & 4.333204724 & -4.000255530 & 1.721974564 \\
$\mathrm{C}$ & 4.916953237 & -2.796053777 & 1.676180189 \\
$\mathrm{C}$ & 4.298473391 & -1.523854985 & 1.560723246 \\
$\mathrm{C}$ & 2.872442486 & -1.476986332 & 1.456132352 \\
$\mathrm{~N}$ & 6.276642278 & -2.526032350 & 1.702100324 \\
$\mathrm{C}$ & 6.427801336 & -1.150451322 & 1.600117881 \\
$\mathrm{~N}$ & 5.264335685 & -0.524242309 & 1.513926893 \\
$\mathrm{H}$ & 1.235557352 & -5.129820160 & 1.590575275 \\
$\mathrm{H}$ & 2.822266355 & -5.951342875 & 1.770212802 \\
$\mathrm{H}$ & 1.243867321 & -2.741751665 & 1.471823235 \\
$\mathrm{H}$ & 7.017684186 & -3.222599791 & 1.803061599 \\
$\mathrm{H}$ & 7.413120451 & -0.670754159 & 1.592630745 \\
$\mathrm{~N}$ & -5.096778890 & -2.284999683 & 1.643333811 \\
$\mathrm{~N}$ & -1.978190398 & -5.221974035 & -1.630534250 \\
$\mathrm{O}$ & 1.196981245 & -1.835642612 & -1.240436776 \\
$\mathrm{~N}$ & -0.321046024 & -3.564780501 & -1.500774990 \\
$\mathrm{C}$ & -0.667549414 & -4.911724442 & -1.619750734 \\
$\mathrm{~N}$ & 0.245846395 & -5.891941124 & -1.701749993 \\
$\mathrm{C}$ & 1.509084300 & -5.450753143 & -1.652334071 \\
$\mathrm{C}$ & 1.968143143 & -4.113489739 & -1.529626138 \\
$\mathrm{C}$ & 0.990712165 & -3.074605911 & -1.418306927 \\
$\mathrm{~N}$ & 2.663071997 & -6.218792075 & -1.681517045 \\
$\mathrm{C}$ & 3.740675096 & -5.351040603 & -1.575024209 \\
$\mathrm{~N}$ & 3.357782847 & -4.086976330 & -1.482544089 \\
$\mathrm{H}$ & -2.745829673 & -4.506867291 & -1.570813918 \\
$\mathrm{H}$ & -2.200789634 & -6.207610887 & -1.764391257 \\
$\mathrm{H}$ & -1.055409661 & -2.821570481 & -1.433569900 \\
$\mathrm{H}$ & 2.696786631 & -7.234651939 & -1.788277765 \\
$\mathrm{H}$ & 4.777463035 & -5.706049753 & -1.569286087 \\
\hline & & Continued & on next page
\end{tabular}


Table S8 - Continued from previous page

\begin{tabular}{cccc}
\hline \hline $\mathrm{N}$ & -5.221373664 & 1.978440090 & -1.628137284 \\
$\mathrm{O}$ & -1.834495123 & -1.196929084 & -1.242441643 \\
$\mathrm{~N}$ & -3.563867095 & 0.321350725 & -1.501016608 \\
$\mathrm{C}$ & -4.910852443 & 0.667867516 & -1.619937222 \\
$\mathrm{~N}$ & -5.890819200 & -0.245527041 & -1.704322603 \\
$\mathrm{C}$ & -5.449423870 & -1.508732566 & -1.657438021 \\
$\mathrm{C}$ & -4.112115194 & -1.967852276 & -1.534660831 \\
$\mathrm{C}$ & -3.073421993 & -0.990510218 & -1.420720172 \\
$\mathrm{~N}$ & -6.217189139 & -2.662822131 & -1.689945174 \\
$\mathrm{C}$ & -5.349361573 & -3.740450552 & -1.585169141 \\
$\mathrm{~N}$ & -4.085406422 & -3.357595761 & -1.490671259 \\
$\mathrm{H}$ & -4.506478825 & 2.746074272 & -1.567297748 \\
$\mathrm{H}$ & -6.206969189 & 2.201255234 & -1.762132572 \\
$\mathrm{H}$ & -2.820728512 & 1.055805739 & -1.432586552 \\
$\mathrm{H}$ & -7.232969459 & -2.696490307 & -1.797893321 \\
$\mathrm{H}$ & -5.704141504 & -4.777331778 & -1.582238886 \\
$\mathrm{~N}$ & 1.978975538 & 5.220106233 & -1.634805665 \\
$\mathrm{O}$ & -1.196861057 & 1.834322124 & -1.242062250 \\
$\mathrm{~N}$ & 0.321677386 & 3.563055511 & -1.503797270 \\
$\mathrm{C}$ & 0.668360634 & 4.909963278 & -1.623956908 \\
$\mathrm{~N}$ & -0.244895482 & 5.890166718 & -1.707039961 \\
$\mathrm{C}$ & -1.508165533 & 5.449266892 & -1.657513681 \\
$\mathrm{C}$ & -1.967494198 & 4.112161486 & -1.533559030 \\
$\mathrm{C}$ & -0.990285859 & 3.073161371 & -1.421030918 \\
$\mathrm{~N}$ & -2.662006144 & 6.217419954 & -1.687920066 \\
$\mathrm{C}$ & -3.739775358 & 5.350048085 & -1.580939044 \\
$\mathrm{~N}$ & -3.357237710 & 4.085940403 & -1.486994472 \\
$\mathrm{H}$ & 2.746339283 & 4.504859980 & -1.574909894 \\
$\mathrm{H}$ & 2.201875732 & 6.205590602 & -1.769887644 \\
$\mathrm{H}$ & 1.056120266 & 2.819742554 & -1.436117796 \\
$\mathrm{H}$ & -2.695496783 & 7.233221893 & -1.795922901 \\
$\mathrm{H}$ & -4.776507895 & 5.705113505 & -1.575861196 \\
$\mathrm{~N}$ & 5.222113437 & -1.980146174 & -1.624562999 \\
$\mathrm{O}$ & 1.834932203 & 1.195546393 & -1.243967054 \\
$\mathrm{~N}$ & 3.564361549 & -0.323161346 & -1.500006770 \\
$\mathrm{C}$ & 4.911518788 & -0.669580392 & -1.617869093 \\
$\mathrm{~N}$ & 5.891414018 & 0.243830637 & -1.702669965 \\
$\mathrm{C}$ & 5.449981927 & 1.507030322 & -1.657752109 \\
$\mathrm{C}$ & 4.112564772 & 1.966157616 & -1.536718265 \\
$\mathrm{C}$ & 3.073905402 & 0.988865768 & -1.421741371 \\
$\mathrm{~N}$ & 6.217705095 & 2.661099107 & -1.690795275 \\
\hline & & Continued & \\
& & & \\
& & &
\end{tabular}


Table S8 - Continued from previous page

\begin{tabular}{cccc}
\hline \hline $\mathrm{C}$ & 5.349804747 & 3.738870472 & -1.587942082 \\
$\mathrm{~N}$ & 4.085784485 & 3.356009917 & -1.494228461 \\
$\mathrm{H}$ & 4.507092143 & -2.747603074 & -1.563543981 \\
$\mathrm{H}$ & 6.207788356 & -2.203010779 & -1.758150434 \\
$\mathrm{H}$ & 2.821217152 & -1.057630163 & -1.431021734 \\
$\mathrm{H}$ & 7.233599062 & 2.694501980 & -1.797548828 \\
$\mathrm{H}$ & 5.704573689 & 4.775733883 & -1.585621390 \\
$\mathrm{Ca}$ & -0.000053605 & -0.000055643 & 0.022745220 \\
\hline
\end{tabular}

